\title{
Zur Lage null- und eindimensionaler Punktmengen
} im $E^{3}$

\author{
von \\ H. G. B othe (Berlin)
}

1. Einleitung. Sind $M$ und $N$ zwei Teilmengen eines euklidischen Raumes $E^{n}$, so heißt $M$ im $E^{n}$ lagetreu in $N$ einbettbar, falls es einen Homöomorphismus von $E^{n}$ auf sich gibt, der $M$ auf eine Teilmenge von $N$ abbildet. Eine Menge $U$ aus einem System $\subseteq$ von Teilmengen eines festen euklidischen Raumes $\mathbb{E}^{n}$ heißt Universalmenge von $\subseteq$ bzgl. der Lage im $E^{n}$, falls sich jede Menge $M$ aus $\subseteq$ im $E^{n}$ lagetreu in $U$ einbetten läßt. In [7] wurden für gewisse Systeme $\subseteq$ Universalmengen bzgl. der Lage angegeben. Hier soll für einige Systeme von Teilmengen des $E^{3}$ bewiesen werden, daß sie keine Universalmengen bzgl. der Lage im $E^{3}$ besitzen. Die beiden Hauptresultate sind:

(a) Zur jeder abgeschlossenen nulldimensionalen Teilmenge $A^{0}$ des $E^{3}$ gibt es eine Cantorsche Teilmenge des $E^{3}$ (d.h. eine zum Cantorschen Diskontinuum homöomorphe Teilmenge des $\left.E^{3}\right)$, die sich im $E^{3}$ nicht lagetreu in $A^{0}$ einbetten läßt.

(b) $Z u$ jeder abgeschlossenen eindimensionalen Teilmenge $A^{1}$ des $E^{3}$ gibt es eine zur Sierpinskischen Universalkurve (siehe z.B. [3]) homöomorphe Teilmenge des $E^{3}$, die sich im $E^{3}$ nicht lagetreu in $A^{1}$ einbetten läßt.

Es sei noch erwähnt, daß beim Beweis von (b) ein in gewissem Sinne überschaubares System $\mathfrak{U}$ von eindimensionalen kompakten Teilmengen des $E^{3}$ konstruiert wird, so daß sich jedes eindimensionale Teilkompaktum von $E^{3}$ im $E^{3}$ lagetreu in eine Menge aus $\mathfrak{U}$ einbetten läßt. Das System $\mathfrak{U}$ ist von der Mächtigkeit des Kontinuums. Aus (a) folgt sofort, daß ein System von nulldimensionalen abgeschlossenen Teilmengen des $E^{3}$, in dem alle Cantorschen Teilmengen von $E^{3}$ vorkommen, niemals eine Universalmenge bzgl. der Lage im $E^{3}$ besitzen kann. Ebenso kann wegen (b) ein System von eindimensionalen abgeschlossenen Teilmengen des $E^{3}$, in dem alle zur Sierpińskischen Universalkurve homöomorphe Teilmengen des $E^{3}$ vorkommen, keine Universalmenge bzgl. der Lage im $E^{z}$ besitzen. Insbesondere enthalten die Systeme aller Cantorschen Teilmengen des $E^{3}$, aller nulldimensionalen kompakten Teilmengen des $E^{3}$, 
aller nulldimensionalen abgeschlossenen Teilmengen des $\mathbb{E}^{3}$, aller eindimensionalen kompakten Teilmengen des $E^{3}$ oder aller eindimensionalen abgeschlossenen. Teilmengen des $E^{3}$ keine Universalmengen bzgl. der Lage im $E^{3}$. Durch das erste dieser Beispiele wird eine Frage von R. H. Bing beantwortet ([5], Seite 441 ).

Die vorliegende Arbeit besteht abgesehen von den vorbereitenden Abschnitten 1 bis 4 aus zwei Teilen. Im ersten Teil wird die Behauptung (a) im zweiten die Behauptung (b) bewiesen. Der zweite Teil ist unabhängig vom ersten Teil lesbar. Bzgl. der verwendeten Bezeichnungen seien die folgenden Verabredungen getroffen: Alle auftretenden Punktmengen und topologischen Räume sind - falls nicht ausdrücklich das Gegenteil gesagt wird - Teilmengen des $E^{3}$. Wird von lagetreuer Einbettung gesprochen, so ist dementsprechend immer die lagetreue Einbettung im $\mathbb{E}^{3}$ gemeint. Wenn von Mannigfaltigkeiten die Rede ist und nichts gegenteiliges gesagt wird, so sind zweidimensionale zusammenhängende geschlossene Mannigfaltigkeiten gemeint. (Da diese nach der soeben getroffenen Verabredung zudem im $E^{3}$ liegen, sind sie orientierbar.) Den $E^{3}$ denken wir auf ein festes Cartesisches Koordinatensystem bezogen, so daß wir seine Punkte als Tripel $\left(\xi_{1}, \xi_{2}, \xi_{3}\right)$ von reellen Zahlen ansehen können. Ein Polyeder ist die Vereinigung aller zu einem (endlichen) simplizialen Komplex gehörenden Simplexe.

2. Homologietheoretische Vorbemerkungen. In diesem $A b-$ schnitt soll der Klarheit und Bequemlichkeit wegen auf einige später verwendete Tatsachen, aus der Homologietheorie hingewiesen werden. Dabei werden wir so einfach und anschaulich wie möglich verfahren und die Definitionen und Sätze nicht allgemeiner aussprechen als es für die Anwendungen in dieser Arbeit nötig ist. Es interessieren uns nur die eindimensionalen Homologieklassen modulo 2 von Polyedern und offenen Mengen (im $E^{3}$ ). Es sei also in diesem Abschnitt mit $A$ stets ein Polyeder oder eine offene Menge bezeichnet.

Unter einer eindimensionalen. Kette von $A$ verstehen wir die Vereinigung von endlich vielen in $A$ enthaltenen Strecken. Der Rand einer solchen Kette ist die Menge der Punkte, von denen eine ungerade Anzahl von Strecken ausgeht. Eine Kette heißt (eindimensionaler) Zylklus, falls ihr Rand leer ist. Um die Summe zweier Zyklen $z_{1}$ und $z_{2}$ zu bilden, stelle man $z_{1}$ und $z_{2}$ so als Vereinigung von Strecken dar, da $\beta$ je zwei aller dabei auftretenden Strecken höchstens Endpunkte gemeinsam haben. Die Summe $z_{1}+z_{2}$ ist dann die Vereinigung aller der Strecken, die in genau einem der beiden Zyklen enthalten sind. Es gilt stets $z+z=0$ (Nullzyklus = leere Menge), und die Menge aller Zyklen von $A$ bildet eine Gruppe $Z(A)$. Eine zweidimensionale Kette $x$ von $A$ ist eine endliche Menge von in $A$ enthaltenen Dreiecken, die paarweise disjunkt sind oder höchstens einen Eckpunkt oder eine Kante gemeinsam haben. Der Rand von $x$ ist die Vereinigung aller Kanten, die zu einer ungeraden Anzahl von Dreiecken aus $x$ gehören. Der Rand einer zweidimensionalen Kette ist stets ein Zyklus. Ein Zyklus heißt in A homolog null, falls er Rand einer in $A$ enthaltenen zweidimensionalen Kette ist. Zwei Zyklen heißen in $A$ homolog, falls ihre Summe in $A$ homolog null ist. Die nullhomologen Zyklen bilden in $Z(A)$ eine Untergruppe $N(A)$ und die Faktorgruppe $H(A)=Z(A) / N(A)$ heißt (eindimensionale) Homologiegruppe von $A$. $\mathrm{Da}$ das doppelte einer Homologieklasse (d.h. eines Elementes aus $H(A)$ ) stets null ist, kann man $H(A)$ als Vektorraum über dem Primkörper der Charakteristik 2 auffassen. Hat $H(A)$ endliche Dimension (was z.B. bei Polyedern stets der Fall ist), so nennen wir diese die (eindimensionale) Bettische Zahl von $A$. Gehören die Zyklen $z_{1}, \ldots, z_{r}$ lauter verschiedenen Homologieklassen von $A$ an und bilden diese Homologieklassen eine Basis von $H(A)$, so nennen wir $z_{1}, \ldots, z_{r}$ eine Homologiebasis von $A$.

Insbesondere ist jedes einfach geschlossene Polygon in $A$ ein Zyklus, wobei zwei in $A$ homotope einfach geschlossene Polygone zur gleichen Homologieklasse gehören. Da jede einfach geschlossene Kurve in $A \mathrm{zu}$ einem einfach geschlossenen Polygon homotop ist, bestimmt eine solche Kurve auf .naheliegende Weise eine Homologieklasse, und wir können diese Kurven ebenfalls als Zyklen ansehen.

Jeder Zyklus ist in $E^{3}$ homolog null. Sind $z_{1}$ und $z_{2}$ zwei disjunkte Zyklen, so kann man in $E^{s}$ eine zweidimensionale Kette $x$ so wählen, da $\beta z_{1}$ der Rand von $x$ ist und $z_{2}$ mit der Vereinigung $\bar{x}$ aller in $x$ enthaltenen Dreiecke nur endlich viele Punkte gemeinsam hat, die alle im Innern von zu $x$ gehörenden Dreiecken liegen und die bei einer passenden Zerlegung von $z_{2}$ in Strecken nicht Endpunkte von solchen Strecken sind. Wir setzen die Versehlingungszaht $\mathfrak{v}\left(z_{1}, z_{2}\right)$ von $z_{1}$ und $z_{2}$ gleich null oder eins je nachdem die Anzahl der gemeinsamen Punkte von $z_{2}$ und $\bar{x}$ gerade oder ungerade ist. Im ersten Fall heißen $z_{1}$ und $z_{2}$ unverschlungen, im zweiten verschlungen. (Man kann zeigen, $\mathrm{da} \mathfrak{b}\left(z_{1}, z_{2}\right)$ von der Auswahl der Kette $x$ unabhängig ist.) Für die Verschlingungszahlen gelten folgende Regeln:

$$
\mathfrak{v}\left(z_{1}, z_{2}\right)=\mathfrak{v}\left(z_{2}, z_{1}\right) ; \quad \mathfrak{v}\left(z_{1}+z_{1}^{\prime}, z_{2}\right)=\mathfrak{v}\left(z_{1}, z_{2}\right)+\mathfrak{v}\left(z_{1}^{\prime}, z_{2}\right)(\bmod 2) ;
$$

ist $z_{1}^{\prime}$ zu $z_{1}$ in $E^{s} \backslash z_{2}$ homolog, so gilt $\mathfrak{v}\left(z_{1}^{\prime}, z_{2}\right)=\mathfrak{v}\left(z_{1}, z_{2}\right)$. Nach der oben gemachten Bemerkung ist es auch sinnvoll von der Verschlingungszahl $\mathfrak{v}\left(C_{1}, C_{2}\right)$ zweier disjunkter einfach geschlossener Kurven $C_{1}$ und $C_{2}$ zu sprechen. Ist $h$ ein Homöomorphismus von $E^{3}$ auf sich, so gilt $\mathfrak{v}\left(O_{1}, C_{2}\right)$ $=\mathfrak{v}\left(h\left(C_{1}\right), h\left(C_{2}\right)\right)$.

Ist $z_{1}, \ldots, z_{r}$ eine Homologiebasis eines Polyeders $P$, so gibt es in $E^{\natural} \backslash P$ Zyklen $z_{1}^{\prime}, \ldots, z_{r}^{\prime}$ mit $\mathfrak{v}\left(z_{i}, z_{j}^{\prime}\right)=\delta_{i j}$ (Kroneckersymbol). Die Zyklen $z_{1}^{\prime}, \ldots, z_{r}^{\prime}$ bilden dann eine Homologiebasis von $E^{3} \backslash P$, und man nennt 
die Basen $z_{1}, \ldots, z_{r}$ und $z_{1}^{\prime}, \ldots, z_{r}^{\prime}$ zueinander dual. Hieraus folgt insbesondere, daß die Homologiegruppen von $P$ und $E^{3} \backslash P$ isomorph sind (Dualitätssatz). (Wegen der dualen Basen siehe [2] XI, insbesondere die Seiten 437 und 443 . Dort werden die Sätze allgemeiner für krumme Seiten 437 und 443. Dort werden die Satze allgemeiner furt.) Als weitere Folgerung aus der Existenz dualer Basen vermerken wir: Ist $z_{1}, \ldots, z_{r}$ eine Homologiebasis von $P$, so sind zwei Zyklen $z$ und $z^{\prime}$ von $E^{3} \backslash P$ genau dann in $E^{3} \backslash P$ homolog, falls für $i=1, \ldots, r$ gilt $\mathfrak{v}\left(z, z_{i}\right)=\mathfrak{v}\left(z^{\prime}, z_{i}\right)$. Hieraus ergibt sich leicht die folgende sräter benötigte Bemerkung:

Es seien $P^{\prime} \subseteq P$ zwei Polyeder und $z_{1}, \ldots, z_{r}$ eine Homologiebasis von $P^{\prime}$, deren $k$ erste $Z$ Zyklen $z_{1}, \ldots, z_{k}(k \leqslant r)$ eine Homologiebasis von $P$ bilden. Dann ist jeder Zyklus $z$ aus $\mathbb{E}^{3} \backslash \boldsymbol{P}^{\prime}$, der mit keinem der Zyjklen $z_{k+1}, \ldots, z_{r}$ verschlungen ist, in $E^{3} \backslash P^{\prime}$ zu einem $Z y$ kilus aus $Z^{3} \backslash P$ homolog.

3. Einbettung in kompakte und abgeschlossene Mengen. Wir formulieren den folgenden eirfachen Satz nur für den $E^{3}$, obwohl er - wie der Beweis zeigt - für beliebige euklidische Räume gilt.

Satz. Ist $A$ eine abgeschlossene $m$-dimensionale Menge, so gibt es eine kompakte $m$-dimensionale Menge $A^{\prime}$ mit der Eigenschaft, daß jede kompakte lagetreu in $A$ einbettbare Menge auch lagetreu in $A^{\prime}$ eingebettet werden kann.

Beweis. Es sei für $r>0$ mit $K(r)$ die durch $\xi_{1}^{2}+\xi_{2}^{2}+\xi_{3}^{2} \leqslant r^{2}$ definierte Vollkugel bezeichnet. Für $n=1,2,3, \ldots$ betrachten wir je einen Homöomorphismus $h_{n}$ von $E^{3}$ auf sich, der $K(n)$ auf $K\left(n^{-1}\right)$ abbildet und setzen

$$
A^{\prime}=\bigcup_{n=1}^{\infty} h_{n}(K(n) \cap A) \cup\{(0,0,0)\} .
$$

$A^{\prime}$ ist sicher kompakt und - wie aus dem dimensionstheoretischen Summensatz folgt - auch $m$-dimensional. Ist $M$ ein lagetreu in $A$ einbettbares Kompaktum und $h$ ein Homöomorphismus von $E^{3}$ auf sich mit $h(M) \subset A$, so liegt $h(M)$ als kompakte Menge für genügend großes $n$ in $K(\bar{n}) \cap A$. Der Homöomorphismus $h_{n} h$ bettet $M$ lagetreu in $A^{\prime}$ ein.

4. Komplexe. Ist $v$ eine positive ganze Zahl und sind $k_{1}, k_{2}, k_{3}$ beliebige ganze Zahlen, so ist die durch

$$
\frac{k_{i}}{v} \leqslant \xi_{i} \leqslant \frac{k_{i}+1}{v} \quad(i=1,2,3)
$$

definierte Menge aller Punkte $\left(\xi_{1}, \xi_{2}, \xi_{3}\right)$ ein achsenparalleler Würfel der Kantenlänge $v^{-1}$. Durchläuft $\left(k_{1}, k_{2}, k_{3}\right)$ bei festem $v$ alle Tripel von ganzen Zahlen, so erhält man ein System von Würfeln, das den ganzen Raum überdeckt und das in Zukunft mit $\mathfrak{W}^{(v)}$ bezeichnet werden soll. Ein $\nu$-Komplex ist eine beliebige endliche Teilmenge von $\mathfrak{W}^{(v)}$. Unter einem Komplex verstehen wir hier stets eine Menge von Würfeln, die für ein gewisses $v$ einen $v$-Komplex bildet. (Simpliziale Komplexe werden wir stets als solche bezeichnen.) Ist $\Omega$ ein Komplex, so sei $[\Omega]$ das Polyeder von $\Re$, d.h. die Vereinigung aller zu $\Omega$ gehörenden Würfel. Unter den $\mu$-Unterteilung $\Omega^{(\mu)}(\mu \geqslant 1$ ganz) eines $\nu$-Komplexes $\Omega$ verstehen wir den $\mu \nu$-Komplex bestehend aus allen in [ $\mathcal{\Omega}$ ] enthaltenen Würfeln aus $\mathfrak{W}^{(\mu))}$. Ist $\mu>1$, so heißt $\mathfrak{\Re}^{(\mu)}$ echte Unterteilung von $\mathfrak{\Omega}$. Offenbar gilt $[\Omega]=\left[\Omega^{(\mu)}\right]$. Zwei Würfel eines Komplexes sollen benachbart genannt werden, falls sie eine gemeinsame Seite haben (eine Seite eines Würfels ist eines der 6 diesen Würfel begrenzenden Quadrate).

\section{DER NULLDIMENSIONALE FALL}

5. Darstellungsfolgen. Wir nennen einen Komplex $\Omega$. einen $M$-Komplex, falls alle Komponenten von $[\mathfrak{R}$ ] (berandete) dreidimensionale Mannigfaltigkeiten sind oder (was damit gleichbedeutend ist) falls alle Komponenten des Randes von [ $\Omega$ ] Mannigfdltigkeiten sind (vgl. die Verabredungen über Bezeichnungen in Abschnitt 1). Dæmit ein Komplex $\mathfrak{R}$ ein $M$-Komplex ist, sind die folgenden Bedingungen notwendig und hinreichend:

(a) Gehört eine Kante zu genau zwei Würfeln aus $\mathfrak{K}$, so haben diese Würfel eine gemeinsame Seite.

(b) Gehört ein Eckpunkt zu genau zwei Würfeln aus $\mathfrak{\Omega}$, so haben diese beiden Würfel eine gemeinsame Seite.

Verstehen wir unter der Umgebung eines $\boldsymbol{\nu}$-Komplexes $\mathfrak{\Omega}$ die Menge der Würfel aus $\mathfrak{S}^{(*)}$, die mit $[\mathfrak{\Omega}]$ einen nicht leeren Durchschnitt haben, so folgt hieraus leicht, daß für jeden Komplex $\Omega$ die Umgebung von $\mathfrak{\Omega}^{(\mu)}$ ein $M$-Komplex ist, wenn nur $\mu$ mindestens gleich 3 ist. Bei einem $M$-Komplex $\mathfrak{R}$ wollen wir mit $\gamma(\mathfrak{K})$ das Maximium der Geschlechter aller Randkomponenten von $[\Omega]$ bezeichnen. Je zwei Komponenten von [ $\Omega$ ] lassen sich dann durch eine in $E^{3} \backslash[\Omega$ ] gelegene Mannigfaltigkeit trennen, deren Geschlecht $\gamma(\boldsymbol{\Omega})$ nicht übersteigt.

Es sei $A$ eine kompakte Menge. Ist $\Re_{1}, \Omega_{2}, \Omega_{3}, \ldots$ eine Folge von $M$-Komplexen mit den Eigenschaften

(a) $\Omega_{i}$ ist Teilkomplex einer echten Unterteitung von $\Omega_{i-1}(i=1,2,3, \ldots)$,

$$
A=\bigcap_{i=1}^{\infty}\left[\Omega_{i}\right],
$$

so soll $\Omega_{1}, \Omega_{2}, \Omega_{3}, \ldots$ Darstellungsfolge von $A$ genannt werden.

Es läßt sich leicht zeigen, daß jedes Kompaktum $A$ eine Darstellungsfolge besitzt. Wir konstruieren nämlich eine Folge $\mathfrak{\Omega}_{1}, \mathfrak{\Omega}_{2}, \mathfrak{\Omega}_{3}, \ldots$, die neben (a) und (b) noch die folgende Bedingung erfüllt: 
$\Omega_{1}$ sei ein beliebiger $M$-Komplex, dessen Polyeder $A$ im Innern enthält. Wir nehmen nun an, $\Omega_{1}, \ldots, \Omega_{j}$ seien bereits so definiert, daß (a) und (c) für $i \leqslant j$ gelten. Ist $\lambda$ eine positive ganze Zahl, so sei $\mathfrak{L}(\lambda)$ die Menge aller Würfel aus $\mathcal{S}_{i}^{(\lambda)}$, die Punkte aus $A$ enthalten. Wählen wir $\lambda$ genügend groß, so ist $[\mathfrak{L}(\lambda)]$ im Innern von $\left[\Omega_{j}\right]$ enthalten. Die Umgebung von $\mathscr{L}(\lambda)^{(3)}$ ist dann ein $M$-Komplex, dessen Polyeder $A$ im Innern enthält und der Teilmenge von $\mathcal{R}^{(8 \lambda)}$ ist. Bezeichnen wir ihn mit $\Omega_{i+1}$, so erfüllt er also die Bedingungen (a) und (c). Daß die so konstruierte Folge $\Omega_{1}, \Omega_{2}, \ldots$ den Durchschnitt $A$ hat, folgt sofort daraus, $\mathrm{da} \beta$ die Durchmesser der in $\Omega_{i}$ enthaltenen Würfel bei wachsendem $i$ gegen 0 streben.

Ist $A$ nulldimensional, so streben die Durchmesser der Komponenten von $\left[\Omega_{i}\right]$ bei wachsendem $i$ gegen 0 . Berücksichtigt man, daß sich je zwei Komponenten von $\left[\Omega_{i}\right]$ durch eine in $E^{3} \backslash\left[\mathcal{\Omega}_{i}\right]$ gelegene Mannigfaltigkeit trennen lassen $\left(\Omega_{i}\right.$ ist ja ein $M$-Komplex), so ergibt sich, daß je zwei Punkte eines nulldimensionalen Kompaktums $A$ durch eine in $E^{3} \backslash A$ gelegene Mannigfaltigkeit getrennt werden können. Für Anwendungen im folgenden Abschnitt bezeichnen wir das Minimum der Geschlechter aller Mannigfaltigkeiten, die in $E^{3} \backslash A$ enthalten sind und $A$ zerlegen, mit $\tau(A)$ (hierbei setzen wir voraus, da $\beta A$ mindestens zwei Punkte enthält). Zur Veranschaulichung sei bemerkt, daß bei der Antoineschen Menge $A$ die $\mathrm{Zahl} \tau(A)$ gleich 1 ist.

6. Beweis modulo Hilfssatz 1. Wir haben zu jeder nulldimensionalen abgeschlossenen Menge $A$ eine Cantorsche Menge $O$ anzugeben, die sich nicht lagetreu in $A$ einbetten läßt. Wegen des Satzes aus $A b$ schnitt 3 dürfen wir dabei voraussetzen, daß $A$ kompakt ist. Zum Beweis ziehen wir folgenden später zu beweisenden Hilfssatz 1 heran.

HIIfSSATZ 1. Ist $\alpha_{1}, \alpha_{2}, \alpha_{3}, \ldots$ eine gegen $+\infty$ strebende Folge von reellen Zahlen und $\beta_{1}, \beta_{2}, \beta_{3}, \ldots$ eine Folge ganzer Zahlen, so gibt es eine Folge $\mathfrak{C}_{1}, \mathfrak{C}_{2}, \mathfrak{C}_{3}, \ldots$ von Komplexen, deren Polyeder $\boldsymbol{C}_{\boldsymbol{i}}=\left[\mathbb{C}_{i}\right]$ folgende Eigenschaften haben:

(a) $C_{i} \subseteq O_{i-1}(i=2,3, \ldots)$.

(b) $C_{1}$ ist zusammenhängend.

(c) $C=\bigcap_{i=1}^{\infty} C_{i}$ ist nulldimensional.

(d) Ist $K$ eine Komponente von $C_{i}$, so enthält $O \cap K$ mindestens zwei Punkte $(i=1,2,3, \ldots)$.

(e) $Z u$ jedem Index $i$ gibt es einen Index $j$, so $d a \beta$

$$
x\left(C_{i}\right) \leqslant \frac{1}{i} \alpha_{j} \text { und } \tau(K \cap C)>\beta_{j}
$$

gilt, wobei $x\left(C_{i}\right)$ die Anzahl der Komponenten von $C_{i}$ bedeutet und $K$ alle Komponenten von $C_{\boldsymbol{i}}$ durchläuft. $(\tau(K \cap C)$ wurde am Ende des vorigen Abschnittes definiert.)

Zunächst sei als Folgerung aus (a), (c) und (d) vermerkt, daß $O$ ein perfektes nulldimensionales Kompaktum also eine Cantorsche Menge ist (vgl. [9], Seite 58).

Es sei nun $A$ ein nulldimensionales Kompaktum und $\Omega_{1}, \Re_{2}, \Re_{3}, \ldots$ eine fest gewählte Darstellungsfolge von $A$. Wir setzen in Hilfssatz 1 $\beta_{j}=\gamma\left(\Omega_{j}\right)\left(\gamma\left(\Omega_{j}\right)\right.$ wurde im letzten Abschnitt definiert) und $\alpha_{j}=\delta_{j}^{-1}$, wobei $\delta_{j}$ das Maximum der Durchmesser aller Komponenten von [ $\left.\mathfrak{\Omega}_{j}\right]$ ist $(j=1,2,3, \ldots)$ und zeigen, daß sich die Menge $C$ dann nicht lagetreu in $A$ einbetten läßt.

$\mathrm{Zu}$ diesem Zwecke nehmen wir an, es gäbe einen Homöomorphismus $h$ von $E^{s}$ auf sich mit $h(C) \subseteq \underline{A}$. Es seien dann $p$ und $q$ zwei verschiedene Punkte aus $C$ und $\varrho$ der Abstand zwischen $h(p)$ und $h(q)$. Mit $i$ bezeichnen wir nun einen beliebigen Index und wählen einen weiteren Index $j$ so, daß die Bedingung (e) efüllt ist. Für jede Komponente $K$ von $C_{i}$ muß dann $h(K \cap C)$ in einer Komponente von [ $\left.\mathcal{\Omega}_{j}\right]$ enthalten sein, denn je zwei Komponenten von $\left[\Omega_{j}\right]$ lassen sich durch eine in $E^{3} \backslash\left[\Omega_{j}\right]$ gelegene Mannigfaltigkeit trennen, deren Geschlecht höchstens gleich

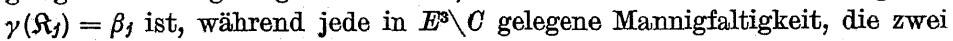
zur gleichen Komponente $K$ von $C_{i}$ gehörende Punkte aus $O$ trennt, mindestens das Geschlecht $\tau(K \cap C)>\beta_{j}$ hat. Es gibt also höchstens $\varkappa\left(C_{i}\right)$ Komponenten von $\left[\Omega_{j}\right]$, die Punkte aus $h(C)$ enthalten. Nun hat jede Komponente von $\left[\Omega_{j}\right]$ höchstens den Durchmesser $\delta_{j}$. Da sich die Punkte $h(p)$ und $h(q)$ nicht durch eine in $E^{3} \backslash h(C)$ gelegene Mannigfaltigkeit vom Geschlecht 0 trennen lassen ( $C_{1}$ ist ja zusammenhängend und daher $\left.\tau\left(C_{1} \cap C\right)=\tau(C)>\beta_{1} \geqslant 0\right)$, folgt hieraus

$$
\varrho \leqslant \varkappa\left(C_{i}\right) \alpha_{j}^{-1} \leqslant \frac{1}{i} \text {. }
$$

Da das für alle $i$ gilt, folgt $\varrho=0$, was wegen $h(p) \neq h(q)$ unmöglich ist.

7. Verkettete Würfel. Es seien $\nabla^{\prime}$ und $\nabla^{\prime \prime}$ die durch

$$
0 \leqslant \xi_{i} \leqslant 7 \quad(i=1,2,3)
$$

bzw. durch

$$
0 \leqslant \xi_{1} \leqslant 7, \quad 0 \leqslant \xi_{2} \leqslant 7, \quad 7 \leqslant \xi_{3} \leqslant 14
$$

definierten Würfel und $\nabla=\nabla^{\prime} \cup V^{\prime \prime}$. In $V$ betrachten wir die Punkte $p_{1}=\left(\frac{3}{2}, \frac{7}{2}, 0\right), \quad p_{2}=\left(\frac{3}{2}, \frac{7}{2}, \frac{17}{2}\right), \quad p_{3}=\left(\frac{11}{2}, \frac{7}{2}, \frac{17}{2}\right), \quad p_{4}=\left(\frac{11}{2}, \frac{7}{2}, 0\right)$, $q_{1}=\left(\frac{7}{2}: \frac{3}{2}, 14\right), \quad q_{2}=\left(\frac{7}{2}, \frac{3}{2}, \frac{11}{2}\right), \quad q_{3}=\left(\frac{7}{2}, \frac{11}{2}, \frac{11}{2}\right), \quad q_{4}=\left(\frac{7}{2}, \frac{11}{2}, 14\right)$ 
and die Streckenzüge

$$
\begin{aligned}
J^{\prime} & =\left[p_{1}, p_{2}\right] \cup\left[p_{2}, p_{3}\right] \cup\left[p_{3}, p_{4}\right], \\
J^{\prime \prime} & =\left[q_{1}, q_{2}\right] \cup\left[q_{2}, q_{3}\right] \cup\left[q_{3}, q_{4}\right] .
\end{aligned}
$$

Schließlich seien $H^{\prime}$ bzw. $H^{\prime \prime}$ die Vereinigungen aller in $V$ gelegenen Würfel aus $\mathfrak{W}^{(1)}$, die $J^{\prime}$ bzw. $J^{\prime \prime}$ schneiden (Abb. 1 ).

Es seien $\delta$ und $\nu$ ganze positive Zahlen. Wir setzen $\mu=v(\delta+2)$ und betrachten die Mengen $\mathfrak{W}^{(\nu)}$ und $\mathfrak{W}^{(\mu)}$. Ist $W$ ein beliebiger Würfel

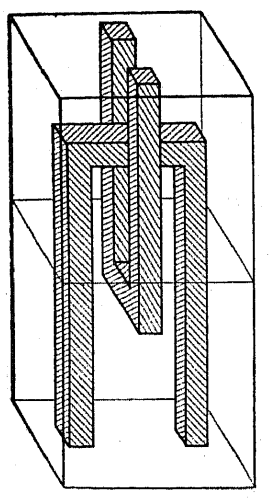

Abb. 1 aus $\mathfrak{W}^{(v)}$, so bezeichnen wir die Vereinigung aller im Innern von $W$ gelegenen Würfel aus $\mathfrak{W}^{(\mu)}$ mit. $W^{*} . W^{*}$ ist also ein mit $W$ konzentrischer Würfel đer Kantenlänge $\nu^{-1}-2 \mu^{-1}=\delta \mu^{-1}$. Sind $W_{1}$ und $W_{2}$ zwei benachbarte Würfel aus $\mathfrak{W}^{(\nu)}$, so gibt es in $\mathfrak{W}^{(\mu)}$ genau $\delta^{2}$ Paare von benachbarten Würfeln $W_{i}^{\prime}, W_{i}^{\prime \prime}\left(i=1, \ldots, \delta^{2}\right)$, die zwischen $W_{1}^{*}$ und $W_{2}^{*}$ liegen, bei denen also $W_{i}^{\prime}$ eine auf der Oberfläche von $W_{1}^{*}$ und $W_{i}^{\prime \prime}$ eine auf der Oberfläche von $W_{2}^{*}$ gelegene Seite hat (siehe Abb. 2). $\mathrm{Zu}$ jedem $i$ wählen wir eine Ähnlichkeitsabbildung $h_{i}$ von $E^{3}$ auf sich, die $V^{\prime}$ in $W_{i}^{\prime}$ und $V^{\prime \prime}$ in $W_{i}^{\prime \prime}$ überführt. Die Menge $h_{i}\left(H^{\prime}\right)$ bezeichnen wir dann. als einen an $W_{1}^{*}$ angebrachten Henkel. Ebenso ist $h_{i}\left(H^{\prime \prime}\right)$ ein an $W_{2}^{*}$ angebrachter Henkel. Auf diese Weise seien für alle Paare $W_{1}, W_{2}$ von benachbarten Würfeln aus $\mathfrak{W}^{(v)}$ sowohl an $W_{1}^{*}$ als auch an $W_{2}^{*}$ Henkel angebracht. Da es zu jedem Würfel 6 benachbarte Würfel gibt, wird also jeder Würfel $W^{*}$ mit $6 \delta^{2}$ Henkeln versehen, und zwar sind an jeder Seite von $W^{*}$ genau $\delta^{2}$ dieser Henkel angebracht.

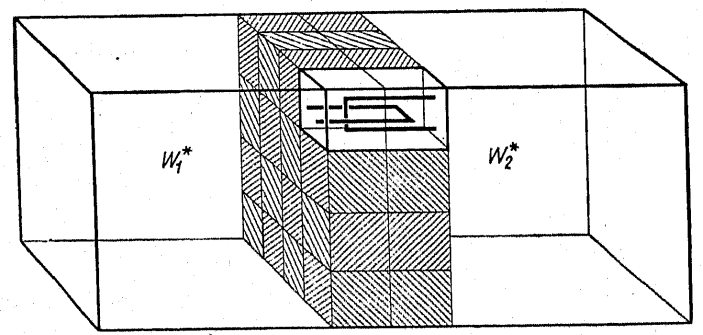

Abb. 2

Ist $W$ ein Würfel aus $\mathfrak{W}^{(v)}$, so sei die Vereinigung von $W^{*}$ mit den $6 \delta^{2}$ angebrachten Henkeln mit $W(\delta)$ bezeichnet. $W(\delta)$ ist dann das Polyeder eines $7 \mu$-Komplexes $(7 \mu=7 \nu(\delta+2))$. Ist $\Re=\left\{W_{1}, \ldots, W_{n}\right\}$ ein $\nu$-Kom- plex, so sei $\Re(\delta)$ der $7 v(\delta+2)$-Komplex, dessen Polyeder gleich $W_{1}(\delta) \cup$ $\cup \ldots \cup W_{n}(\delta)$ ist. $[\Re(\delta)]$ zerfällt in die Komponenten $W_{1}(\delta), \ldots, W_{n}(\delta)$.

Wir betrachten nun einen Würfel $W$ aus $\mathfrak{M}^{(\nu)}$, den zugehörigen Würfel $W^{*}$ und einen in $W(\delta)$ an $W^{*}$ angebrachten Henkel $H$. Die Vereinigung $H \cup W^{*}$ ist im topologischen Sinne ein Volltorus. Ein einfach geschlossenes Polygon, das in diesem Volltorus genau einmal umläuft, wollen wir eine Seele des Henkels $H$ nennen (siehe Abb. 3).

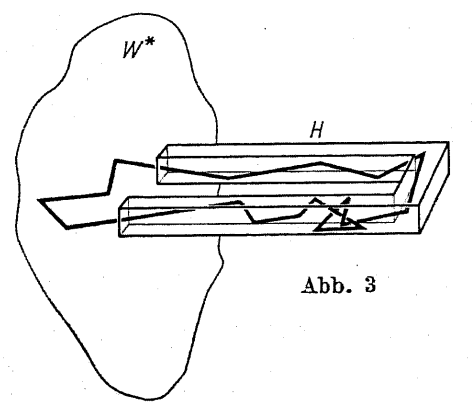

Nun seien $W_{1}$ und $W_{2}$ zwei benachbarte Würfel aus. $\mathfrak{W}^{(*)}$. Mit $H_{i}^{\prime}$ bezeichnen wir die an der $W_{2}^{*}$ zugekehrten Seite von $W_{1}^{*}$ angebrachten Henkel und mit $h_{i}$ eine Ähnlichkeitsabbildung, die $H^{\prime}$ in $H_{i}^{\prime}$ überführt $\left(i=1, \ldots, \delta^{2}\right)$. Die Mengen $h_{i}\left(H^{\prime \prime}\right)=H_{i}^{\prime \prime}$ sind dann die Henkel der $W_{1}^{*}$ zugewandten Seite von $W_{2}^{*}$. Wir nennen $H_{i}^{\prime}$ mit $H_{i}^{\prime \prime}$ verschlungen; sprechen wir von den Henkeln, durch die $W_{1}(\delta)$ und $W_{2}(\delta)$ miteinander verkettet sind, so meinen wir damit diese Henkel $H_{i}^{\prime}$ und $H_{i}^{\prime \prime}\left(i=1, \ldots, \delta^{2}\right)$. Diese Bezeichnungen sind anschaulich klar und werden durch folgende Relationen zwischen den Verschlingungszahlen von Seelen gerechtfertigt: Sind $C_{i}^{\prime}$ Seelen von $H_{i}^{\prime}$ und $C_{i}^{\prime \prime}$ Seelen von $H_{i}^{\prime \prime}\left(i=1, \ldots, \delta^{2}\right)$, so gilt für die Verschlingungszahlen (siehe Abschnitt 2)

$$
\mathfrak{v}\left(C_{i}^{\prime}, C_{j}^{\prime \prime}\right)=\delta_{i j} \quad \text { (Kroneckersymbol). }
$$

Zum Beweis betrachte man zuerst die Seelen $C_{i}^{\prime}=h_{i}\left(J^{\prime} \cup\left[p_{4}, p_{1}\right]\right)$ und $C_{i}^{\prime \prime}=h_{i}\left(J^{\prime \prime} \cup\left[q_{4}, q_{1}\right]\right)$, für die die Relationen sofort einzusehen sind und beachte dann, daß je zwei Seelen des gleichen Henkels in $W_{1}(\delta)$ bzw. $W_{2}(\delta)$ homolog sind.

Es soll jetzt eine wichtige Eigenschaft der Komplexe $\Omega(\delta)$ bewiesen werden. Dazu führen wir folgende Bezeichnung ein: Sind $W_{1}$ and $W_{2}$ zwei benachbarte Würfel aus irgendeinem Komplex mit den Zentren $z_{1}$ bzw. $z_{2}$ und ist $M$ eine Mannigfaltigkeit, die weder $z_{1}$ noch $z_{2}$ enthält, so sagen wir, daß $M$ zwischen $z_{1}$ und $z_{2}$ hindurchgeht, falls die Punkte $z_{1}$ und $z_{2}$ in verschiedenen Komponenten von $\left(W_{1} \cup W_{2}\right) \backslash M$ liegen. 
Hufssatz 2. Ist $\Omega$ ein Komplex, $\delta$ eine positive Zahl und $M$ eine polyederartige Mannigfaltigkeit, die zwischen den Zentren $z_{1}$ und $z_{2}$ zweier benachbarter $W$ ürfel $W_{1}, W_{2}$ aus $\Omega$ hindurchgeht, die aber niemals zwischen den Zentren zweier benachbarter Würfel aus $\Re(\delta) \cap \mathfrak{\Omega}^{(7(\delta+2))}$ hindurchgeht und keines dieser Zentren enthält, so hat MI mindestens das Geschlecht $\delta^{2}$.

Beweis. $M$ ist ein endliches Polyeder, und wir dürfen voraussetzen, daß $M$ eine Triangulation $T$ mit folgenden Eigenschaften besitzt: (a) Kein Eckpunkt von $T$ liegt in einer Ebene, die eine Seite von $W_{1}$ oder $W_{2}$ enthält. (b) Keine Kante von $T$ schneidet eine Gerade, die eine Kante von $W_{1}$ oder $W_{2}$ enthält. (c) Kein Dreieck von $T$ enthält einen Eckpunkt von $W_{1}$ oder $W_{2}$. (Das läßt sich durch eine beliebig kleine Verrückung von $M$ erreichen, bei der $M$ alle vorausgesetzten Eigenschaften beibehält.) Die Komponenten von $M \cap\left(W_{1} \cup W_{2}\right)$ sind dann berandete zweidimensionale Mannigfaltigkeiten, deren Randkurven auf der Oberfläche von $W_{1} \cup W_{2}$ liegen.

Es seien $H_{i}^{\prime}$ und $H_{i}^{\prime \prime}\left(i=1, \ldots, \delta^{2}\right)$ die Henkel, durch die $W_{1}(\delta)$ und $W_{2}(\delta)$ miteinander verkettet sind, wobei die Numerierung wieder so gewählt ist, daß jeweils $H_{i}^{\prime}$ mit $H_{i}^{\prime \prime}$ verschlungen ist. Da $M$ niemals $z w i-$ schen den Zentren zweier Würfel aus $\mathcal{K}(\delta) \cap \Re^{(7(\delta+2))}$ hindurchgeht, gibt es Seelen $O_{i}^{\prime}$ von $H_{i}^{\prime}$, die $M$ nicht schneiden und mit $z_{1}$ in derselben Komponente von $\left(W_{1} \cup W_{2}\right) \backslash M$ liegen. Ebenso gibt es Seelen $C_{i}^{\prime \prime}$ von $H_{i}^{\prime \prime}$, die zu $M$ fremd sind und mit $z_{2}$ in derselben Komponente von $\left(W_{1} \cup W_{2}\right) \backslash M$ liegen.

Es sei nun $N$ eine Komponente von $M \cap\left(W_{1} \cup W_{2}\right)$, die in $W_{1} \cup W_{2}$ die Punkte $z_{1}$ und $z_{2}$ voneinander trennt. Sind $R_{1}, \ldots, R_{r}$ die Randkurven von $N$, so kann man paarweise disjunkte zweidimensionale polyederartige Zellen $D_{1}, \ldots, D_{r}$ finden, so daß $D_{i} \cap\left(W_{1} \cup W_{2}\right)=R_{i}$ wird, und die Menge $M^{\prime}=N \cup D_{1} \cup \ldots \cup D_{r}$ ist eine zweidimensionale geschlossene Mannigfaltigkeit, deren Geschlecht nicht größer als das von $M$ ist. Die Seelen $C_{i}^{\prime}$ und $C_{i}^{\prime \prime}$ liegen in verschiedenen Komponenten von $B^{3} \backslash M^{\prime}$, und aus den Relationen

$$
\mathfrak{v}\left(C_{i}^{\prime}, C_{j}^{\prime \prime}\right)=\delta_{i j}
$$

zwischen den Verschlingungszahlen folgt, daß die durch die Polygone $O_{i}^{\prime}$ und $C_{j}^{\prime \prime}$ repräsentierten Homologieklassen der Menge $E^{3} \backslash M^{\prime}$ unabhängig sind. Wäre nämlich etwa $O_{1}^{\prime}$ in $E^{9} \backslash M^{\prime}$ zu $\sum_{i=2}^{\delta^{2}} \alpha_{i} O_{i}^{\prime}+\sum_{j=1}^{\delta^{2}} \beta_{j} O_{j}^{\prime \prime}$ homolog, so müßte $O_{1}^{\prime}$ zu $\sum_{i=2}^{\delta^{2}} \alpha_{i} C_{i}^{\prime}$ homolog sein, da ja die $O_{j}^{\prime \prime}$ in der anderen Komponente von $E^{s} \backslash M^{\prime}$ liegen. Diese Homologie gälte dann auch in der $C_{1}^{\prime}$ enthaltenden Komponente von $E^{3} \backslash M^{\prime}$ und erst recht in $E^{3} \backslash C_{1}^{\prime \prime}$. Es wäre also

$$
\mathfrak{v}\left(C_{1}^{\prime}, O_{1}^{\prime}\right)=\mathfrak{v}\left(\sum_{i=2}^{\delta^{2}} \alpha_{i} C_{i}^{\prime}, C_{1}^{\prime \prime}\right)=\sum_{i=2}^{\delta^{2}} \alpha_{i} \mathfrak{v}\left(C_{i}^{\prime}, C_{1}^{\prime \prime}\right)=0 .
$$

Die Bettische Zahl von $E^{3} \backslash M^{\prime}$ kann also nicht kleiner als $2 \delta^{2}$ sein. Nach dem Alexanderschen Dualitätssatz (siehe Abschnitt 2) ist die Bettische Zahl von $M^{\prime}$ ebenfalls nicht kleiner als $2 \delta^{2}$. Das Geschlecht von $M^{\prime}$ und damit auch das von $M$ muß also mindestens gleich $\delta^{2}$ sein. (Die Bettische Zahl einer Mannigfaltigkeit ist ja gleich dem doppelten Geschlecht.)

Es sei noch bemerkt, daß bei ungeradem $\delta$ alle Zentren von Würfeln aus $\Re$ auch Zentren gewisser Würfel aus $\mathcal{R}(\delta)$ sind.

8. Beweis des Hilfssatzes 1. Nach diesen Vorbereitungen können wir nunmehr den Hilfssatz 1 beweisen. Es sei also $\alpha_{1}, \alpha_{2}, \alpha_{3}, \ldots$ eine gegen $+\infty$ konvergierende Folge reeller Zahlen und $\beta_{1}, \beta_{2}, \beta_{3}, \ldots$ eine Folge ganzer Zahlen. Die Folge $\mathfrak{V}_{1}, \mathfrak{V}_{2}, \mathfrak{V}_{3}, \ldots$ soll schrittweise konstruiert werden. Zuerst sei $\mathfrak{C}_{1}$ ein Komplex, der nur aus einem einzigen Würfel besteht. Die weiteren Komplexe $\mathfrak{c}_{2}, \mathfrak{c}_{3}, \ldots$ definieren wir, indem wir sukzessive eine Folge $\delta_{1} \leqslant \delta_{2} \leqslant \ldots$ von ungeraden ganzen Zahlen angeben und

$$
\mathfrak{C}_{i+1}=\mathbb{C}_{i}\left(\delta_{i}\right) \cap \mathbb{C}_{i}^{\left(7\left(\delta_{i}+2\right)\right)}
$$

setzen. Wir nehmen jetzt an, $\mathfrak{C}_{1}, \ldots, \mathfrak{C}_{i}(i \geqslant 1)$ und damit $\delta_{1}, \ldots, \delta_{i-1}$ seien bereits festgelegt. Um $\delta_{i}$ zu definieren, betrachten wir den kleinsten Index $j$, für den $\varkappa\left(C_{i}\right) \leqslant \alpha_{j} / i$ wird $\left(\varkappa\left(C_{i}\right)\right.$ ist die Anzahl der Komponenten des Polyeders $C_{i}=\left[\mathbb{C}_{i}\right]$ ). Sodann sei $\delta_{i}$ eine beliebige ungerade Zahl, die größer als $\beta_{j}$ und im Falle $i \geqslant 2$ nicht kleiner als $\delta_{i-1}$ ist.

$\mathrm{Da} B$ die Polyeder $C_{1}, C_{2}, \ldots$ der so konstruierten Komplexe $\mathfrak{C}_{1}, \boldsymbol{C}_{2}, \ldots$ die im Hilfssatz 1 mit (a), (b), (c) und (d) bezeichneten Forderungen erfüllen, ist unmittelbar klar. Weiterhin folgt aus der Konstruktion, dab es zu jedem Index $i$ einen Index $j(i)$ gibt, für den gilt

$$
\varkappa\left(C_{i}\right) \leqslant \frac{1}{i} \alpha_{j(i)}, \quad \delta_{i}>\beta_{j(i)} .
$$

Es kommt jetzt darauf an, für die Folgende $C_{1}, C_{2}, \ldots$ auch die Eigenschaft (e) nachzuweisen. Es sei dazu $i$ ein Index und $K$ eine Komponente von $C_{i}$. Wir setzen $j=j(i)$ und beweisen dann die geforderten Ungleichungen

$$
x\left(G_{i}\right) \leqslant \frac{1}{i} \alpha_{j}, \quad \tau(K \cap C)>\beta_{j} .
$$

Die erste dieser beiden Ungleichungen ist unmittelbare Folgerung aus der Definition von $j(i)$. Um die zweite Ungleichung zu verifizieren ist zu zeigen, daß jede in $E^{3} \backslash(K \cap C)$ gelegene Mannigfaltigkeit $M$, die $K \cap C$ zerlegt, mindestens das Geschlecht $\beta_{j}+1$ hat. Nach dem Bingschen Approximationssatz für Mannigfaltigkeiten (siehe [4]) dürfen wir $M$ als Polyeder voraussetzen. Für $l \geqslant i$ sei $\Omega_{l}$ der Teilkomplex von $\mathfrak{C}_{l}$, 
der aus allen in $\boldsymbol{K}$ enthaltenen Würfeln aus $\mathfrak{C}_{l}$ besteht. Offenbar ist $K=\left[\Re_{i}\right]$ und $K \cap C=\bigcap_{i=i}^{\infty}\left[\Omega_{l}\right]$. Weiterhin gilt

$$
\Re_{l+1}=\Re_{l}\left(\delta_{l}\right) \cap \Re_{l}^{\left(7\left(\delta_{l}+2\right)\right)} .
$$

Da die Zahlen $\delta_{1}, \delta_{2}, \delta_{3}, \ldots$ alle ungerade sind, ist die Menge aller Zentren von Würfeln aus $\Omega_{l}(l \geqslant i)$ eine dichte Teilmenge von $K \cap 0$. Hieraus und aus der Tatsache, daß $M$ von $K \cap O$ einen positiven Abstand hat, folgt die Existenz eines größten Index $l$, für den $M$ zwischen den Zentren zweier Würfel aus $\Omega_{l}$ hindurchgeht. Ein Blick auf den Hilfssatz 2 zeigt, daß $M$ mindestens das Geschlecht $\delta_{l}^{2}$ haben muß. Aus $\delta_{l} \geqslant \delta_{i}>\beta_{j}$ folgt dann das ersehnte Resultat.

\section{DER EINDIMENSIONALE FALI}

9. Knoten. Es sei $\mathfrak{R}$ die Menge aller zahmen einfach geschlossenen orientierten Kurven. (Eine einfach geschlossene Kurve heißt orientiert, falls auf ihr ein Durchlaufungssinn ausgezeichnet ist. Zwei Kurven, die sich durch ihre Orientierung unterscheiden, werden als verschieden angesehen.) Man nennt zwei Kurven $C_{1}$ und $C_{2}$ aus $\Omega$ gleichverlenotet, falls es einen Homöomorphismus von $E^{3}$ auf sich gibt, der die Orientierung von $E^{3}$ erhält und $O_{1}$ auf $C_{2}$ abbildet (unter Berücksichtigung der auf diesen Kurven ausgezeichneten Orientierungen). Dor von einer Kurve $O$ aus $\Omega$ repräsentierte Knoten $\langle\boldsymbol{C}\rangle$ ist die Menge aller mit $O$ gleichverknoteten Kurven aus $\Omega$. Jeder Knoten enthält ein einfach geschlossenes orientiertes Polygon; ja mehr noch, ist $p(t)(0 \leqslant t \leqslant 1 ; p(0)=p(1))$ eine Parameterdarstellung einer Kurve aus $\mathfrak{R}$ und $\varepsilon$ eine positive Zahl, so gibt es ein dazu gleichverknotetes Polygon mit einer Parameterdarstellung $q(t)(0 \leqslant t \leqslant 1 ; q(0)=q(1))$, bei der der Abstand $\varrho(p(t), q(t))$ stets kleiner als $\varepsilon$ ist (das folgt aus [11] Satz 1 ).

Um die Ergebnisse der beiden Arbeiten [13] und [14] anwenden zu können, betrachten wir auch die Knotentheorie in der dreidimensionalen Sphäre $S^{3}$, wie sie diesen Arbeiten zugrundeliegt. Es sei $T^{4}$ das im vierdimensionalen euklidischen Raum $E^{4}$ von den Punkten $0=(0,0,0,0)$, $p=(1,0,0,0), q=(0,1,0,0), r=(0,0,1,0), s=(0,0,0,1)$ aufge spannte Simplex, $S^{3}$ der Rand von $T^{4}$ und $T^{3}$ das von $o, p, q, r$ aufgespannte dreidimensionale Simplex. Den Raum $E^{3}$ denken wir vermöge $\left(\xi_{1}, \xi_{2}, \xi_{3}\right) \rightarrow\left(\xi_{1}, \xi_{2}, \xi_{3}, 0\right)$ in $D^{4}$ eingebettet.

Wir betrachten die Menge $\Omega_{8}$ aller einfach geschlossenen orientierten Polygone in $S^{3}$. Ähnlich wie oben nennen wir zwei Polygone $P_{1}$ und $P_{2}$ aus $\Omega_{s}$ in $S^{3}$ gleichverknotet, falls es einen semilinearen Homöomorphismus von $S^{3}$ auf sich gibt, der die Orientierung von $S^{s}$ erhält und $P_{1}$ auf $P_{\mathbf{2}}$ abbildet (unter Berücksichtigung der auf diesen Polygonen ausgezeichneten Orientierungen). (Ein Homöomorphismus $h$ von einem Polyeder auf ein Polyeder heißt semitinear, falls es (endliche) Simplizialzerlegungen der Polyeder gibt, bei denen $h$ eine simpliziale Abbildung ist.) Der von einem Polygon $P$ aus $\Omega_{s}$ in $S^{3}$ repräsentierte Knoten $\langle P\rangle$ ist die Menge aller mit $P$ gleichverknoteten Polygone aus $\Omega_{s}$. Ist $x$ ein Knoten in $E^{3}$, so läßt sich $x$ durch ein in $T^{3}$ gelegenes Polygon $P$ repräsentieren. Durch $P$ wird auch ein Knoten $\varkappa^{\prime}$ von $S^{3}$ repräsentiert, und die $\mathrm{Zu}$ ordnung $x \rightarrow x^{\prime}$ ist eine eineindeutige Abbildung der Menge aller Knoten von $E^{3}$ auf die Menge aller Knoten von $S^{3}$ (das folgt aus [12] Satz (6.1) und [8] Seite 37 f.). Vermöge dieser Zuordnung lassen sich die Ergebnisse aus [13] und [14], auf die wir in diesem und im rächsten Abschnitt verweisen werden, leicht auf die hier betrachteten Knoten im $E^{3}$ übertragen.

Es sei $Z^{3}$ ein zur dreidimensionalen Vollkugel homöomorphes Polyeder mit dem Rand $S^{2}$ und $B$ ein in $Z^{3}$ verlaufender orientierter Strekkenzug, der mit $S^{2}$ nur seinen Anfangs- und Endpunkt gemeinsam hat. Wir nennen dann $B$ eine Sehne von $Z^{3}$. Ist $B^{*}$ ein in $S^{2}$ gelegener Bogen, der $B \mathrm{zu}$ einem einfach geschlossenen Polygon $B \cup B^{*}$ erछänzt, so ist $B \cup B^{*}$ durch die Orientierung von $B$ eberfalls orientiert, und der Knoten $\left\langle B \cup B^{*}\right\rangle$ in $E^{\mathbf{3}}$ hängt nicht von der Auswahl des Bogens $B^{*}$ ab (siehe [13] Satz 1). Wir nennen $\left\langle B \cup B^{*}\right\rangle$ den von $B$ in $Z^{3}$ repräsentierten Knoten. Zwei Sehnen $B_{2}$ und $B_{1}$ von $Z^{\mathbf{3}}$ repräsentieren in $Z^{\mathbf{3}}$ genau dann den gleichen Kroten, falls es einen orientierungserhaltenden Homöomorphismus $h$ von $Z^{3}$ auf sich gibt, der $B_{1}$ unter Berücksichtigung der Orientierung auf $B_{2}$ abbildet. Haben $B_{1}$ und $B_{2}$ den gleichen Arfangspunkt und den gleichen Endpunkt, so kann man $h$ so wählen, daß alle Punkte des Randes $S^{2}$ von $Z^{3}$ bei $h$ festbleiben (siehe [13], Seite 15).

Es seien $Z_{1}^{3}$ und $Z_{2}^{3}$ zwei zur Vollkugel homöomorphe Polyeder mit den Rändern $S_{1}^{2}$ bzw. $S_{2}^{2}$, so daß $Z_{1}^{3} \cap Z_{2}^{3}=S_{1}^{2} \cap S_{2}^{2}=D$ ein zur Kreisscheibe homöomorphes Polyeder ist. Ferner seien $B_{1}$ und $B_{2}$ zwei Sehnen von $Z_{1}^{3}$ bzw. $Z_{2}^{3}$, wobei der Endpunkt von $B_{1}$ mit dem Arfangspunkt von $B_{2}$ übereinstimmt und im Innern von $D$ liegt, während der Anfangspunkt von $B_{1}$ und der Endpunkt von $B_{2}$ außerhalb $D$ liegen. Dann ist $B=B_{1} \cup B_{2}$ eine Sehne von $Z^{3}=Z_{1}^{3} \cup Z_{2}^{3}$. Repräsentieren $B_{1}$ bzw. $B_{2}$ in $Z_{1}^{3}$ bzw. $Z_{2}^{3}$ den Knoten $\varkappa_{1}$ bzw. $\varkappa_{2}$, so hängt der von $B$ in $Z^{3}$ repräsentierte Knoten $x_{\text {nur }}$ von $x_{1}$ und $x_{2}$ ab und wird als Produkt $\varkappa_{1} \varkappa_{2}$ von $x_{1}$ und $x_{2}$ bezeichnet (vgl. [13] insbesondere Seite 22). Diese Multiplikation von Knoten macht die Menge aller Knoten zu einer regulären kommutativen Halbgruppe, in der der triviale Knoten (d.h. der von Rande eines in $E^{3}$ gelegenen Dreiecks repräsentierte Knoten) die Rolle des Einselementes einnimmt. Aus der Konstruktion des Produktes zweier Knoten ergibt sich leicht die folgende Bemerkung: 


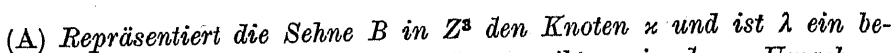
liebiger Knoten und $\varepsilon$ eine positive Zahl, so gibt es in der $\varepsilon$-Umgebung von $B$ eine Sehne $B^{\prime}$ von $Z^{3}$, deren Anfangs- und Endpunlt mit dem $A n$ fangs- bzw. Endpunkt von $B$ zusammenfallen und die in $Z^{3}$ den Knoten $x \cdot \lambda$ repräsentiert.

Mit $W_{e}$ soll in Zukunft stets der durch $0 \leqslant \xi_{i} \leqslant 1(i=1,2,3)$ definierte Einheitswïrfel bezeichnet werden. Die Menge der Punkte aus $W_{e}$, die nicht in der durch $\frac{1}{4}<\xi_{i}<\frac{3}{4}(i=1,2)$ definierten Menge liegen, bilden ein zum Volltorus homöomorphes Polyeder V. Unter einem Vollring $R$ verstehen wir ein Polyeder in $S^{\mathbf{3}}$, das mittels eines (bei passenden Triangulationen von $R$ und $V$ ) semilinearen Homöomorphismus auf $V$ abgebildet werden kann. Ein im Innern eines Vollringes $R$ gelegenes einfach geschlossenes orientiertes Polygon $P$ heißt Secle von $R$, falls es eine semilineare Abbildung $f$ des Einheitswürfels $W_{e}$ auf $R$ mit folgenden Eigenschaften gibt:

(a) Es gitt $f(p)=f(q)$ genau dann, wenn $p=\left(0, \xi_{2}, \xi_{3}\right)$ und $q=$ $=\left(1, \xi_{2}, \xi_{3}\right)$.

(b) Die Strecke $\left[\left(0, \frac{1}{2}, \frac{1}{2}\right],\left(1, \frac{1}{2}, \frac{1}{2}\right)\right]$ wird bei $f$ auf $P$ abgebildet. (Siehe hierzu [14], §6, insbesondere Hilfssatz 2.)

Jeder Vollring besitzt eine Seele, und die von den Seelen eines festen Vollringes in $S^{3}$ repräsentierten Knoten unterscheiden sich höchstens durch ihre Orientierung (siehe [14], §6 insbesondere Hilfssatz 1). Repräsentiert eine Seele eines Vollringes den Knoten $x$, so sagen wir, daß $x$ von diesem Vollring repräsentiert wird. Zu jedem Knoten gibt es dann einen repräsentierenden Vollring (siehe $[14] \S 10$ Hilfssatz 1 ). Ein einfach geschlossenes Polygon $P$ heißt trivial in einem Vollring $R$ gelegen, falls $P$ Seele von $R$ ist oder falls es in $R$ ein $P$ enthaltendes zur Vollkugel homöomorphes Polyeder gibt. Wir nennen einen Knoten $\varkappa$ aus $S^{s}$ schwächer verknotet als einen Knoten $\lambda$ aus $S^{3}$ und schreiben $x<\lambda$, falls $x$ der triviale Knoten ist und $x \neq \lambda$ gilt oder falls $x$ nicht der triviale Knoten ist und es einen $x$ repräsentierenden Vollring gibt, der ein $\lambda$ repräsentierendes Polygon nicht trivial im Innern enthält. Die Relation < führt in die Menge aller Knoten von $S^{3}$ eine teilweise Ordnung ein, d.h. sie erfïllt die folgenden Bedingungen: (a) es gilt niemals $x<x$; (b) aus $x_{1}<x_{2}$ und $x_{2}<x_{3}$ folgt $x_{1}<x_{3}$. (Siehe hierzu [14], $\S 13$, wo die Definitionen nur unwesentlich von den hier ausgesprochenen abweichen.) Gilt $x=\lambda$ oder unterscheiden sich die Knoten $x$ und $\lambda$ nur durch die Orientierung oder gilt $x<\lambda$, so sagen wir, daß $x$ nicht stärker als $\lambda$ verknotet ist und schreiben $x \leqslant \lambda$. Die Relation $\leqslant$ hat folgende Eigenschaften: $\left(a^{\prime}\right) x \leqslant x ;\left(b^{\prime}\right)$ aus $x_{1} \leqslant x_{2}$ und $x_{2} \leqslant x_{3}$ folgt $x_{1} \leqslant x_{3} ;\left(c^{\prime}\right)$ aus $x_{1} \leqslant x_{2}$ und $x_{2} \leqslant x_{1}$ folgt, daß sich $x_{1}$ und $x_{2}$ höchstens durch die Orientierung unterscheiden. Die Relation $x \leqslant \lambda$ besteht genau dann, wenn es einen $x$ repräsentierenden Vollring $R$ und ein $\lambda$ repräsentierendes Polygon $P$ gibt, das im Innern von $R$ nicht aber in einem in $R$ enthaltenen zur Vollkugel homöomorphen Polyeder liegt. Schließlich notieren wir noch die folgende Bemerkung (siehe [14], § 14):

(B) Ist $\lambda$ nicht der triviale Knoten, so gilt stets $x<\varkappa \lambda$.

Die so in die Menge aller Knoten von $S^{3}$ eingeführten Relationen $<$ und $\leqslant$ übernehmen wir gemäß der oben angegebenen eineindeutigen Beziehung in die Menge aller Knoten von $E^{3}$.

10. Röhren. Es sei $W$ ein Würfel aus $\mathfrak{M}^{(\ell)}$ (siehe Abschn. 4) und $\nu>2$ eine ganze Zahl. Wir betrachten einen Teilkomplex $\Re=\left\{W_{1}, W_{2}\right.$, $\left.\ldots, W_{r}\right\}$ der $v$-Unterteilung $\{W\}^{(v)}$ des nur aus dem Würfel $W$ bestehenden Komplexes $\{W\}$ mit folgenden Eigenschaften:

(a) Es ist $r \geqslant 3$, und alle Würfel $W_{1}, \ldots, W_{r}$ sind paarweise verschieden.

(b) $W_{1}$ und $W_{r}$ haben je genau eine auf der Oberfläche von $W$ gelegene Seite $D$ bzw. $D^{\prime}$, während $W_{2}, \ldots, W_{r-1}$ im Innern von $W$ liegen.

(c) $W_{i}$ und $W_{i+1}$ haben eine gemeinsame Seite $(i=1, \ldots, r-1)$.

(d) Ist $|i-j|>2$, so gilt $W_{i} \cap W_{j}=0$. (Für $|i-j|=2$ können $W_{i}$ und $W_{h}$ höchstens eine gemeinsame Kante haben.)

Unter diesen Voraussetzungen heißt das Polyeder [ $\mathfrak{R}]$ eine Röhre von $W$. Genauer: $R$ ist eine Röhre von $W$, falls es einen Komplex $\Re$ mit den oben angegebenen Eigenschaften gibt, dessen Polyeder gleich $R$ ist. Offenbar ist dieser Komplex $\Re$ durch $R$ eindeutig bestimmt, er soll der zur Röhre $R$ gehörende Komplex genannt werden. Die beiden Quadrate $D$ und $D^{\prime}$ heißen Deckel von $R$ (es ist stets $D \cap D^{\prime}=0$ ). Sind $m_{1}, \ldots, m_{r}$ die Mittelpunkte von $W_{1}, \ldots, W_{r}$ und sind $a$ bzw. $b$ die Mittelpunkte von $D$ bzw. $D^{\prime}$, so nennen wir den Streckenzug

$$
\left[a, m_{1}\right] \cup\left[m_{1}, m_{2}\right] \cup \ldots \cup\left[m_{r-1}, m_{r}\right] \cup\left[m_{r}, b\right]
$$

die Mittellinie der Röhre $R$. Offenbar ist diese Mittellinie durch $R$ eindeutig bestimmt. Versehen wir die Mittellinie von $R$ mit einer Orientierung, so wird sie zu einer Sehne von $W$. Eine Röhre von $W$, auf deren Mittellinie eine Orientierung ausgezeichnet ist, soll orientierte Röhre von $W$ heißen. Die Würfel des zu einer orientierten Röhre gehörenden Komplexes sind auf natürliche Weise angeordnet. Wir nennen den Deckel einer orientierten Röhre $R$, der den Anfangspunkt der orientierten Mittellinie von $R$ enthält, den Eingang von $R$. Eine orientierte Röhre von $W$ repräsentiert in $W$ einen Knoten, nämlich den von ihrer orientierten Mittellinie in $W$ repräsentierten Knoten. Ist ein beliebiger Knoten vorgegeben, so kann man in $W$ offenbar eine diesen Knoten repräsentierende orientierte Röhre finden, die sich sogar so wählen läßt, daß die End- 
punkte ihrer Mittellinie die Mittelpunkte zweier vorgegebener Seiten von $W$ sind.

Für spätere Anwendungen formulieren wir jetzt eine Reihe von Sätzen über Röhren. Es sei $W$ ein Würfel aus $\mathfrak{W}^{(e)}, R$ eine orientierte Röhre in $W$ mit dem Eingang $D$, die in $W$ den Knoten $x$ repräsentieren möge und $L$ die Randkurve von $D$. Mit $V$ bezeichnen wir die abgeschlossene Hülle von $W \backslash R$ und mit $P$ ein einfach geschlossenes orientiertes Polygon in $E^{3} \backslash \nabla$.

(A) Es gilt $\varkappa \leqslant\langle P\rangle$ oder es gibt in $E^{3} \backslash \nabla$ ein $P$ enthaltendes zur Vollkugel homöomorphes Polyeder.

(B) Ist $P$ mit $L$ verschlungen, so gilt $\varkappa \leqslant\langle P\rangle$. (Bei homologietheoretischen Betrachtungen - also insbesondere bei Aussagen über Verschlingungen - lassen wir stets etwa vorhandene Orientierungen unberücksichtigt.)

(C) Es sei jetzt $R$ in $W$ unverknotet (d.h. $\varkappa$ sei trivial). $M i t ~ R=\left\{W_{1}\right.$, ..., $\left.W_{r}\right\}$ beztichnen wir den zu $R$ gehörenden Komplex, dessen Würfel in der natürlichen Reihenfolge numeriert seien. Schließlich setzen wir $W_{i} \cap W_{i+1}$ $=S_{i}(i=1, \ldots, r-1), S_{0}=D$ und $S_{r}=D^{\prime}\left(D^{\prime}\right.$ ist der von $D$ verschiedene Deckel von $R$ ). Ist dann für $l=1, \ldots, r C_{l}$ eine Sehne von $W_{l}$, die im Mittelpunkt von $S_{l-1}$ beginnt und im Mittelpunkt von $S_{l}$ endet und die in $W_{e}$ den Knoten $x_{l}$ repräsentiert, so ist $B=C_{1} \cup \ldots \cup C_{r}$ (mit der Orientierung von $S_{0}$ nach $S_{r}$ ) eine Sehne von $W$, die in $W$ den Knoten $\varkappa_{1} \ldots x_{r}$ repräsentiert.

(D) Ist $R$ in $W$ unverknotet und ist $C$ eine auf dem Rande. von $R$ verlaujende Sehne von $W$, die die beiden Deckel von $R$ verbindet, so ist auch $C$ in $W$ unverknotet.

Beim Beweis dieser Sätze dürfen wir offenbar voraussetzen, daß $W$ und $P$ und damit alle in Frage kommenden Mengen in dem im letzten Abschnitt eingeführten Simplex $T^{3}=E^{3} \cap S^{s}$ liegen. Die in $S^{3}$ gebildete abgeschlossene Hülle von $S^{3} \backslash V$ ist dann ein Vollring $R^{*}$, der in $S^{3}$ den Knoten $x$ repräsentiert. Um das einzusehen zerlege man $R^{*}$ durch die beiden Deckel $D$ und $D^{\prime}$ von $R$ in die beiden zur Vollkugel homöomorphen Polyeder $R$ und $\overline{S^{3} \backslash W}$ und bemerke, daß die Mittellinie von $R$ eine in $R$ unverknotete Sehne ist (siehe [14] Seite 157f.). Um (A) zu beweisen, nehmen wir $\varkappa \nless\langle P\rangle$ an. Dann muß $P$ trivial in $R^{*}$ liegen ohne Seele von $R^{*}$ zu sein. Es gibt also in $S^{3} \backslash V$ ein zur Vollkugel homöomorphes Polyeder, das $P$ enthält. Hierauf ist es leicht, ein ebensolches Polyeder in $E^{3} \backslash \nabla$ zu konstruieren. Zum Beweis von (B) vermerken wir, daß $L$ Meridiankurve von $R^{*}$ ist und wegen [14], Seite 172, Hilfssatz 2 die Ordnung von $R^{*}$ bzgl. $P$ nicht 0 sein kann. Die Behauptung folgt dann sofort aus (A) und [14], Seite 171, Satz 1. Wir beweisen (C): Auf Grund der Definition des Produktes von Knoten repräsentiert die Sehne $B$ in $R$ den Knoten $x_{1} \ldots x_{r}$. Man wende nun den in [14] auf Seite 162 stehenden Hilfssatz 5 an. Die Behauptung (D) schließlich ist eine leichte Folgerung aus [14], Seite 160, Hilfssatz 3.

11. Die Röhren $R_{e}(x)$ und die Mengen $A\left(x^{*}, \mu^{*}\right)$. Zu jedem Knoten $\varkappa$ wählen wir im Einheitswürfel $W_{e}$ eine orientierte Röhre $\boldsymbol{R}_{\epsilon}(x)$, die in $W_{e}$ den Knoten $*$ repräsentiert und deren orientierte Mittellinie den Anfangspunkt $a=\left(0, \frac{1}{2}, \frac{1}{2}\right)$ und den Endpunkt $b=\left(1, \frac{1}{2}, \frac{1}{2}\right)$ besitzt. Diese getroffene Wahl wird in Zukunft beibehalten, so daß bei vorgegebenem $\approx$ die orientierte Röhre $R_{e}(x)$ wohldefiniert ist. Wir führen noch eine Reihe weiterer Bezeichnungen ein:

$v(x)$ ist die Zahl, für die der zu $R_{e}(x)$ gehörende Komplex $v(x)$-Komplex ist.

$D_{e}(x)$ ist der Eingang von $R_{e}(x)$.

$L_{e}(x)$ ist die Randkurve des Quadrates $D_{e}(x)$.

$\nabla_{e}(x)$ ist die abgeschlossene Hülle von $W_{e} \backslash R_{e}(x)$.

Es sei nun $W$ ein Würfel aus $\mathfrak{B}^{(n)}$. Wir betrachten den Komplex $\{W\}^{(\mathbf{s})}=\left\{W_{1}, \ldots, W_{27}\right\}$, wobei die Numerierung der Würfel so vorgenommen sei, daß $W_{1}$ und $W$ das gleiche Zentrum besitzen, während jeder der Würfel $W_{2}, \ldots, W_{7}$ mit $W_{1}$ eine gemeinsame Seite hat. Für $i=2, \ldots, 7$ wählen wir eine Ähnlichkeitsabbildung $f_{i}$ des Raumes $E^{3}$ auf sich, die $W_{e}$ so auf $W_{i}$ abbildet, da $\beta$ die durch $\xi_{1}=0$ bestimmte Seite von $W_{*}$ in die gemeinsame Seite von $W_{i}$ und $W_{1}$ übergeht. Wir setzen:

$$
W(x)=\bigcup_{i=2}^{7} f_{i}\left(\nabla_{e}(x)\right) \cup \bigcup_{i=8}^{27} W_{i}
$$

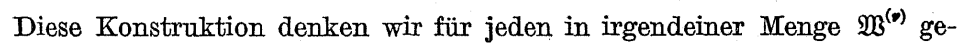
legenen Würfel $W$ und jeden Knoten $*$ ausgeführt, so da $B$ bei Vorgabe von $W$ und $x$ die Menge $W(x)$ eindeutig bestimmt ist. Gehört $W$ zu $\mathfrak{B}^{(*)}$, so ist $W(x)$ das Polyeder eines $3 \cdot v \cdot v(x)$-Komplexes. Für einen beliebigen $\nu$-Komplex $\Re=\left\{W_{1}, \ldots, W_{s}\right\}$ und einen beliebigen Knoten $\varkappa$ definieren wir $\Omega(\varkappa)$ als denjenigen $3 \cdot v \cdot v(\varkappa)$-Komplex, dessen Polyeder mit $\bigcup_{i=1}^{s} W_{i}(x)$ übereinstimmt.

$\mathrm{Zu}$ jeder Folge $x_{1}, \ldots, \varkappa_{n}$ von Knoten und jeder Folge $\mu_{1}, \ldots, \mu_{n}$ von positiven ganzen Zahlen definieren wir einen Komplex $\mathfrak{A}\left(\varkappa_{1}, \ldots, \varkappa_{n}\right.$; $\left.\mu_{1}, \ldots, \mu_{n}\right)$ indem wir setzen:

$$
\begin{aligned}
& \mathfrak{A}=\left\{W_{e}\right\}, \\
& \mathfrak{I}\left(\varkappa_{1} ; \mu_{1}\right)=\left(\mathfrak{A}^{\left(\mu_{1}\right)}\right)\left(\varkappa_{1}\right), \\
& \mathfrak{U}\left(\varkappa_{1}, \varkappa_{2} ; \mu_{1}, \mu_{2}\right)=\left(\mathfrak{A}\left(x_{1} ; \mu_{1}\right)^{\left(\mu_{2}\right)}\right)\left(x_{2}\right) \text {, } \\
& \ldots \ldots \ldots \ldots \ldots \ldots \ldots \ldots \\
& \mathfrak{A}\left(\varkappa_{1}, \ldots, x_{n} ; \mu_{1}, \ldots, \mu_{n}\right)=\left(\mathfrak{A}\left(x_{1}, \ldots, x_{n-1} ; \mu_{1}, \ldots, \mu_{n-1}\right)^{\left(\mu_{n}\right)}\right)\left(\varkappa_{n}\right)
\end{aligned}
$$


Das Polyeder $\left[\mathfrak{A}\left(x_{1}, \ldots, x_{n} ; \mu_{1}, \ldots, \mu_{n}\right)\right]$ bezeichnen wir mit $A\left(x_{1}, \ldots, x_{n}\right.$; $\left.\mu_{1}, \ldots, \mu_{n}\right)$. Ist $a^{*}=\left(a_{1}, a_{2}, \ldots\right)$ irgeneine unendliche Folge so sei stets $\left.\mu_{1}^{*}, \ldots, \mu_{n}\right)$. von Knoten und eine unendliche Folge, $\mu^{*}$ positiver ganzer Zahlen. Es sei dann

$$
A\left(\varkappa^{*} ; \mu^{*}\right)=\bigcap_{i=1}^{\infty} \mathcal{A}\left(x_{i}^{*} ; \mu_{i}^{*}\right) .
$$

Zur Abkürzung benützen wir noch folgende Bezeichnungen:

Es ist also

$$
\begin{aligned}
A\left(x_{1}, \ldots, x_{n} ; 1,1, \ldots, 1\right) & =A\left(x_{1}, \ldots, x_{n}\right), \\
\mathfrak{A}\left(x_{1}, \ldots, x_{n} ; 1,1, \ldots, 1\right) & =\mathfrak{A}\left(x_{1}, \ldots, x_{n}\right), \\
A\left(x^{*} ; 1,1, \ldots\right) & =\boldsymbol{A}\left(x^{*}\right) .
\end{aligned}
$$

$$
\mathfrak{U}\left(x_{1}, \ldots, x_{i+1}\right)=\left(\mathfrak{U}\left(x_{1}, \ldots, x_{i}\right)\right)\left(x_{i+1}\right) .
$$

Wie aus [6] Abschn. 4 hervorgeht, sind die Mengen $A\left(x^{*} ; \mu^{*}\right)$ eindimensionale Kontinua. Wenn man berücksichtigt, daß $A\left(x^{*} ; \mu^{*}\right)$ die Kanten aller zu einem der Komplexe $\mathfrak{A}\left(x_{i}^{*} ; x_{i}^{*}\right)$ gehörenden Würfel enthält, erkennt man leicht, daß $A\left(x^{*} ; \mu^{*}\right)$ keine offene in die Ebene einbettbare Teilmenge enthält und damit (siehe [3]) zur Sierpińskischen Universalkurve homöomorph sein muß.

Zum SchluB dieses Abschnittes führen wir noch die folgende Bemerkung über die Polyeder $W(x)$ an:

(A) Sind $m$ und $m^{\prime}$ die Mittelpunlete zweier Seiten des Würfels W, so gibt es in $W$ eine zu $W(x)$ fremde Sehne, die $m$ mit $m^{\prime}$ verbindet und in $W$ den Knoten $x \cdot x^{\prime}$ repräsentiert, wobei $x^{\prime}$ der durch Umkehrung der Orientierung aus $x$ hervorgehende Knoten ist.

12. Einbettung in $A\left(x^{*} ; \mu^{*}\right)$. Ziel dieses Abschnittes ist es, den. folgenden Satz zu beweisen:

SATz. Zu jedem eindimensionalen Kompaktum A kann man eine Knotenfolge $x^{*}$ und eine Folge $\mu^{*}$ von positiven ganzen Zahlen so bestimmen, daß sich $A$ lagetreu in $A\left(x^{*} ; \mu^{*}\right)$ einbetten läßt, wobei diese Einbettung sogar so vorgenommen werden kann, daß das Bild von $A$ ganz im Innern des Einheitswürfels $W_{e}$ liegt.

Beweis. (1) Es sei $W$ Würfel eines $\nu$-Komplexes, $a^{\prime}$ und $b^{\prime}$ die Mittelpunkte zweier gegenüberliegender Seiten von $W$ und $\varphi$ eine Ähnlichkeitsabbildung von $E^{3}$ auf sich, die den Einheitswürfel $W_{0}$ auf $W$ abbildet, wobei die Punkte $a=\left(0, \frac{1}{2}, \frac{1}{2}\right)$ und $b=\left(1, \frac{1}{2}, \frac{1}{2}\right)$ in $a^{\prime}$ bzw. $b^{\prime}$ übergehen. Weiter sei $X$ ein Kompaktum, das von $a^{\prime}$ und $b^{\prime}$ mindestens den Abstand $(6 v)^{-1} \sqrt{2}$ hat und $B$ eine von $a^{\prime}$ nach $b^{\prime}$ verlaufende Sehne von $W$, die zu $X$ punktfremd ist. Ist dann $x$ der von $B$ in $W$ repräsen- tierte Knoten und $\lambda$ ein beliebiger Knoten, so gibt es einen Homöomorphismus $f$ von $E^{3}$ auf sich mit folgenden Eigenschaften:

$$
f(p)=p \quad \text { für alle } \quad p \in E^{3} \backslash W .
$$

(Hieraus folgt $f(W)=W$ und $\varrho(f(q), q)<\sqrt{3} v^{-1}$ für alle Punkte q.)

$$
f(X) \cap W \underline{C} \varphi\left(\nabla_{e}(x \cdot \lambda)\right) .
$$

Um das zu beweisen, darf man sich offenbar auf den Fall $W=W_{e}$, $v=1, a^{\prime}=a, b^{\prime}=b, \varphi=$ Identität beschränken. $B$ sei jetzt also eine von $a$ nach $b$ verlaufende Sehne von $W_{e}$, die zu $X$ fremd ist und in $W_{e}$ den Knoten $*$ repräsentiert. Nach der Bemerkung (A) aus Abschnitt 9 gibt es auch eine zu $X$ fremde von $a$ nach $b$ laufende Sehne von $W_{e}$, die in $W_{e}$ den Knoten $* \cdot \lambda$ repräsentiert. Wir können also einen Homöomorphismus $f_{1}$ von $W_{e}$ auf sich finden, der alle Randpunkte von $W_{e}$ festläßt und diese Sehne auf die orientierte Mittellinie der Röhre $R_{e}(\varkappa \cdot \lambda)$ abbildet. Die auf dem Rande von $W_{e}$ gelegenen Punkte der Menge $f_{1}(X)$ sind, da sie von $a$ und $b$ mindestens den Abstand $\sqrt{2} / 6$ haben, in $V_{e}(x \cdot \lambda)$ enthalten. Es ist daher leicht, einen Homöomorphismus $f_{2}$ von $W_{c}$ auf sich zu finden, der die Randpunkte von $W_{e}$ festläßt und $f_{1}(X)$ aus $R_{e}(x \cdot \lambda)$ ,herausdrückt". Setzt man $f(p)=f_{2} f_{1}(p)$ für $p \in W_{e}$ und $f(p)=p$ sonst, so hat $f$ alle geforderten Eigenschaften.

(2) Es sei $\Re=\left\{W_{1}, \ldots, W_{r}\right\}$ ein $\nu$-Komplex, und für $1 \leqslant i \leqslant r$ bezeichnen wir mit $W_{i}^{\prime}$ den mit $W_{i}$ konzentrischen Würfel der 3-Unterteilung $\Re^{(3)}$ von $\Omega$. Ist dann $X$ ein eindimensionales in $[\Omega]$ enthaltenes Kompaktum, das keinen inneren Punkt eines der Würfel $W_{i}^{\prime}$ enthält und das von den Mittelpunkten der Seiten der Würfel aus $\boldsymbol{\Omega}^{(3)}$ mindestens den Abstand $(6 \cdot 3 \cdot v)^{-1} \sqrt{2}$ hat, so gibt es einen Knoten $\psi$ und einen Homöomorphismus $g$ von $E^{3}$ auf sich mit den folgenden Eigenschaften:

$$
\begin{aligned}
& g(p)=p \quad \text { für } \quad p \in E^{3} \backslash[\Re] . \\
& g(W)=W \quad \text { für alle Würfel } W \text { aus } \Omega^{(3)} . \\
& g(X) \subseteq[\Re(\varkappa)] .
\end{aligned}
$$

Wegen (a) und (b) gilt $\varrho(g(q), q) \leqslant(3 v)^{-1} \sqrt{3}$ für alle Punkte $q$.

Zum Beweis wählen wir in jedem Würfel $W$ aus $\Omega^{(3)}$, der mit einem der ,Zentralwürfel" $W_{i}^{\prime}$ genau eine Seite $S$ gemeinsam hat, eine zu $X$ fremde Sehne $B$, die vom Mittelpunkt der Seite $S$ zum Mittelpunkt der $S$ gegenüberliegenden Seite von $W$ verläuft. ( $\mathrm{Da} X$ ein eindimensionales Kompaktum ist, muß nach [1], Seite 208, die Menge $W \backslash X$ zusammenhängend sein, so daß Sehnen der genannten Art tatsächlich existieren.) Es sei $*$ das Produkt aller von diesen Sehnen $B$ in den zugehörigen Wür- 
feln $\dot{W}$ repräsentierten Knoten. Ist $W$ einer dieser Würfel, so gilt $W \cap[\mathcal{R}(x)]=\varphi_{W}\left(\nabla_{e}(x)\right)$, wobei $\varphi_{W}$ eine Ähnlichkeitsabbildung von $E^{3}$ auf sich ist, die $W_{e}$ auf $W$ so abbildet, daß der Punkt $\left(0, \frac{1}{2}, \frac{1}{2}\right)$ in den Mittelpunkt der Seite $S$ von $W$ übergeht. Dann geht $[\Omega(x)]$ aus $[\Omega]$ dadurch hervor, daß man aus [ $\mathcal{\Omega}$ ] alle inneren Punkte der Würfel $W_{i}^{\prime}(i=1, \ldots, r)$ entfernt und die oben mit $W$ bezeichneten Würfel durch die Mengen $\varphi_{W}\left(V_{e}(x)\right)$ ersetzt. Wir wenden jetzt auf jeden dieser Würfel $W$, seine Sehne $B$ und den Knoten $*$ die unter (1) bewiesene Tatsache an und erhalten so Homöomorphismen, die jeweils nur die Punkte eines der Würfel $W$ verrücken und dabei den in $W$ gelegenen Teil von $X$ in die Menge $\varphi_{W}\left(\nabla_{e}(x)\right)=W \cap[\mathcal{R}(x)]$ hineindrücken. Das Produkt $g$ aller dieser Homöomorfhismen hat dann offenbar die geforderten Eigenschaften.

(3) Es sei $\Omega=\left\{W_{1}, \ldots, W_{r}\right\}$ ein $\nu$-Komplex und $W_{i}^{\prime}$ wieder der mit $W_{i}$ konzentrische Würfel aus $\mathcal{\Omega}^{(3)}(1 \leqslant i \leqslant r)$. Ist dann $X$ ein in [ $\mathcal{\Omega}$ ] enthaltenes eindimensionales Kompaktum, so gibt es einen Homöomorphismus $h$ von $E^{\text {s }}$ auf sich mit folgenden Eigenschaften:

$$
\begin{aligned}
h([\mathcal{\Omega}]) & =[\Re] . \\
h(X) \cap W_{i}^{\prime} & =0 \quad(i=1, \ldots, r) .
\end{aligned}
$$

(c) Ist $m$ der Mittelpunkt einer Seite eines Würfels aus $\mathfrak{\Re}^{(3)}$ so hat $h(\dot{X})$. von $m$ mindestens den Abstand $(6 \cdot 3 \cdot v)^{-1} \sqrt{2}$.

(d) Für alle Punkte $p$ aus $E^{3}$ gilt

$$
\varrho(h(p), p) \leqslant v^{\prime} \bar{v}^{-1} .
$$

Da $X$ in $[\Re]$ nirgends dicht liegt, kann man zunächst einen Homöomorphismus $h_{1}$ von $E^{\mathbf{s}}$ auf sich finden, der außerhalb [ $\Omega$ ] die identische Abbildung ist, jeden Würfel aus $\mathcal{R}$ unter Festhaltung der Randpunkte auf sich abbildet und die Würfel $W_{i}^{\prime}$ von Punkten der Menge $X$ befreit. Sodann schlage man um jeden Seitenmittelpunkt $m$ eines jeden Würfels aus $\mathcal{R}^{(3)}$ eine abgeschlossene Vollkugel $K(m)$ vom Radius $(6 \cdot 3 \cdot v)^{-1} \sqrt{2}$. Diese Kugeln sind paarweise disjunkt und die einzige Seitenfläche eines Würfels aus $\mathfrak{B}^{(3 v)}$ die von der Kugel $K(m)$ geschnitten wird, ist die Seite $S_{m}$ mit dem Zentrum $m$. Da $h_{1}(X)$ ein eindimensionales Kompalztum ist, kann $h_{1}(X) \cap S_{m}$ in $S_{m}$ nirgends dicht liegen. Indem man für jedes $m$ die in $K(m)$ gelegenen Punkte von $h_{1}(X)$ von einem nahe bei $m$ gelegenen Punkt der Menge $S_{m} \backslash h_{1}(X)$ ausgehend geradlinig aus $K(m)$ herausdrückt, erhält man leicht einen Homöomorphismus $h_{2}$ von $E^{3}$ auf sich, der die Würfel aus $\mathfrak{W}^{(3 v)}$ auf sich abbildet und alle Kugeln $K(m)$ von Punkten aus $h_{1}(X)$ befreit. Der Homöomorphismus $h=h_{2} h_{1}$ hat dann alle geforderten Eigenschaften.
(4) Wendet man (3) und (2) nacheinander an, so ergibt sich:

Ist $\Omega$ ein $v$-Komplex und $X$ ein in [ $[$ ] gelegenes eindimensionales Kompaktum, so gibt es einen Knoten $\varkappa$ und einen Homöomorphismus $l$ von $E^{3}$ auf sich mit folgenden Eigenschaften:

$$
\begin{aligned}
l(X) \subseteq[\Omega(x)], & \\
\varrho(l(p), p) & <3 v^{-1} \quad \text { für alle } p .
\end{aligned}
$$

(5) Wird eine Folge $l_{1}, l_{2}, l_{3}, \ldots$ von Homöomorphismen des $E^{3}$ auf sich schrittweise konstruiert, so da $\beta$ zuerst $l_{1}$, dann $l_{2}$, dann $l_{3}$ usw. festgelegt werden, so kann man jeweils nach Festlegung von $l_{1}, \ldots, l_{i}$ eine positive Zahl $\varepsilon_{i}$, die von $l_{1}, \ldots, l_{i}$ abhängt, mit folgenden Eigenschaften auswählen: Gilt für alle $i$ und alle Punkte $p$ stets $\varrho\left(l_{i+1}(p), p\right) \leqslant \varepsilon_{i}$, so konvergiert die Folge $k_{i}=l_{i} l_{i-1} \ldots l_{1}$ von Homöomorphismen gegen einen Homöomorphismus $k$ von $E^{3}$ auf sich.

Diese Bemerkung, deren Beweis man etwa in [5], Seite 443, findet, gestattet es, den Beweis des Satzes rasch zum Ende zu führen. Zunächst sei $l_{1}$ ein Homöomorphismus von $E^{3}$ auf sich, der $A$ ins Innere von $W_{e}$ überführt. Sodann sei $\varepsilon_{1}$ gemäß der soeben gemachten Bemerkung festgelegt. Wir wählen $\mu_{1} \geqslant 3 \varepsilon_{1}^{-1}$ und wenden (4) auf den Fall $\Omega=\left\{W_{e}\right\}^{\left(\mu_{1}\right)}$, $X=l_{1}(A)$ an. So erhalten wir einen Knoten $x_{1}$ und einen Homöomorphismus $l_{2}$ der die Punkte von $E^{3}$ um höchstens $\varepsilon_{1}$ verrückt und $l_{1}(A)$ in $A\left(\varkappa_{1} ; \mu_{1}\right)$ abbildet. Nun legen wir $\varepsilon_{2}$ fest und wählen $\mu_{2}$ so groß, daß die Kantenlänge der Würfel aus $\left(\mathfrak{H}\left(\varkappa_{1} ; \mu_{1}\right)\right)^{\left(\mu_{2}\right)}$ nicht größer als $\frac{1}{3} \varepsilon_{2}$ wird. Eine Anwendung von (4) auf den Fall $\mathfrak{K}=\left(\mathfrak{H}\left(\varkappa_{1} ; \mu_{1}\right)\right)^{\left(\mu_{2}\right)}, X=l_{2} l_{1}(A)$ ergibt einen Knoten $x_{2}$ und einen Homöomorphismus $l_{3}$, der keinen Punkt um mehr als $\varepsilon_{2}$ verrückt und der $l_{2} l_{1}(A)$ in $A\left(x_{1}, x_{2} ; \mu_{1}, \mu_{2}\right)$ abbildet. So fortfahrend erhalten wir Folgen $x^{*}=\left(x_{1}, x_{2}, \ldots\right), \mu^{*}=\left(\mu_{1}, \mu_{2}, \ldots\right)$ und $l_{1}, l_{2}, \ldots$, wobei die Homöomorphismen $k_{i}=l_{i} \ldots l_{1}(i=1,2, \ldots)$ gegen einen Homöomorphismus $k$ von $E^{3}$ auf sich konvergieren, der $\boldsymbol{A}$ in $A\left(x^{*} ; \mu^{*}\right)$ einbettet. Wählen wir die Zahlen $\varepsilon_{1}, \varepsilon_{2}, \ldots$ genügend klein, so liegt $k(A)$ im Innern von $W_{e}$.

13. Ein Hilfssatz. Ziel dieses Abschnittes ist es, den folgenden Hilfssatz zu beweisen:

HIIfSSatz 3. Für jede endliche Knotenfolge $\varkappa_{1}, \ldots, \varkappa_{n}$ sei $h\left(\varkappa_{1}, \ldots, x_{n}\right)$ die Anzahl der eindimensionalen Homologieklassen des Polyeders $A\left(x_{1}, \ldots, x_{n}\right)$ d.h. die Ordnung der Gruppe $H\left(A\left(\varkappa_{1}, \ldots, \varkappa_{n}\right)\right)$ (vgl. Abschn. 2). Ist dann $\left(x_{1}, x_{2}, \ldots\right)=x^{*}$ eine unendliche Folge von Knoten mit der Eigenschaft $\varkappa_{1}<\varkappa_{2}<\ldots$ und sind $C_{1}, \ldots, C_{t}$ in $E^{3} \backslash A\left(\varkappa^{*}\right)$ gelegene zahme einfach geschlossene orientierte Kurven, so folgt aus $t>h\left(x_{s}^{*}\right)$ und $\varkappa_{s+1} \nless\left\langle C_{1}\right\rangle, \ldots$, $\varkappa_{\beta+1} \nless\left\langle C_{t}\right\rangle, d a \beta$ zwei der Kurven $C_{1}, \ldots, C_{t}$ (als eindimensionale Zyklen betrachtet) in $E^{3} \backslash A\left(x^{*}\right)$ homolog sein müssen (s ist hierbei irgendein fester Index). (Wegen der Bezeichnungen siehe Abschn. 11.) 
Beim Beweis dieses Hilfssatzes wird sich die folgende später angewandte Bemerkung ergeben:

In $A\left(x^{*}\right)$ gibt es ein einfach geschlossenes Polygon $L$ mit der Eigenschaft, daß jede in $E^{3} \backslash A\left(x^{*}\right)$ gelegene zahme einfach geschlossene orientierte Kurve, die mit $L$ verschlungen ist, verknotet sein muß.

Ehe wir den Hilfssatz 3 beweisen, führen wir zunächst einige $\mathrm{Be}$ zeichnungen ein und beweisen dann drei mit (A), (B) bzw. (C) bezeichnete Behauptungen.

Wie im Hilfssatz sei $x^{*}=\left(x_{1}, x_{2}, \ldots\right)$ eine unendliche Knotenfolge mit der Eigenschaft $x_{1}<x_{2}<\ldots$ Wir setzen zur Ablürzung

$$
\begin{gathered}
\mathfrak{N}_{1}=\left\{W_{e}\right\}, \quad \mathfrak{A}_{i+1}=\mathfrak{A}\left(x_{i}^{*}\right) \quad(i=1,2, \ldots), \\
A_{i}=\left[\mathfrak{A}_{i}\right] \quad(i=1,2, \ldots), \\
A=A\left(x^{*}\right)=\bigcap_{i=1}^{\infty} A_{i} .
\end{gathered}
$$

Bei dieser Definition wird $\mathfrak{A}_{i+1}=\mathfrak{A}_{i}\left(\varkappa_{i}\right)$ (vgl. Abschn. 11). Die Würfel aus $\mathfrak{H}_{i}^{(s)}$, die mit keinem Würfel aus $\mathfrak{Y}_{i}$ konzentrisch sind und die nicht in $A_{i+1}$ enthalten sind, sollen mit $W_{1}^{i}, \ldots, W_{r(i)}^{i}$ bezeichnet werden. Erinnert man sich an den im Abschnitt 11 beschriebenen Übergang von $\mathfrak{A}_{4}$ zu $\mathfrak{A}_{i+1}=\mathfrak{U}_{i}\left(\varkappa_{i}\right)$, so sieht man, daß jeder Würfel $W$ aus $\mathfrak{A}_{i}$ genau 6 der Würfel $W_{k}^{i}$ enthält; es sind das gerade die in $W$ enthaltenen Würfel aus $\mathfrak{A}_{i}^{(3)}$, die mit dem zu $W$ konzentrischen Würfel $W^{*}$ aus $\mathfrak{U}_{i}^{(8)}$ genau eine Seite gemeinsam haben. Beim Ü̉bergang von $A_{i}$ zu $A_{i+1}$ werden die Würfel $W_{k}^{i}$ durch Mengen $\varphi_{k}^{i}\left(\nabla_{e}\left(x_{i}\right)\right)$ ersetzt, wobei $\varphi_{k}^{i}$ eine Ähnlichkeitsabbildung von $E^{3}$ auf sich ist, die $W_{e}$ auf $W_{k}^{i}$ abbildet und dabei die durch $\xi_{1}=0$ definierte Seite von $W_{e}$ in die gemeinsame Seite von $W_{k}^{i}$ und $W^{*}$ überführt. Es gilt also

$$
W_{k}^{i} \cap A_{i+1}=\varphi_{k}^{i}\left(V_{e}\left(x_{i}\right)\right) \quad(i=1,2, \ldots ; k=1, \ldots, r(i)) .
$$

Wir führen folgende Bezeichnungen ein:

$$
\begin{array}{ll}
R_{k}^{i}=\varphi_{k}^{i}\left(R_{e}\left(x_{i}\right)\right), & V_{k}^{i}=\varphi_{k}^{i}\left(\nabla_{e}\left(x_{i}\right)\right), \\
D_{k}^{i}=\varphi_{k}^{i}\left(D_{e}\left(x_{i}\right)\right), & L_{k}^{i}=\varphi_{k}^{i}\left(L_{e}\left(x_{i}\right)\right) .
\end{array}
$$

(Siehe Abschn. 11.) Die $D_{k}^{i}$ sind paarweise disjunkte Quadrate mit den Rändern $\boldsymbol{L}_{k}^{i}$.

(A) Es sei $K$ ein zur Vollkugel homöomorphes Polyeder, $S^{2}$ der Rand von $K, J$ ein auf $S^{2}$ gelegenes einfach geschlossenes Polygon und $F$ ein zur Kreisscheibe homöomorphes Polyeder mit der Randkurve $J$, das mit Ausnahme von $J$ ganz im Innern von $K$ liegt. Ferner sei $P$ ein im Innerm von $K$ verlaufendes einfach geschlossenes orientiertes Polygon, das $F$ schneidet. Die Menge $K \backslash F$ zerfällt dann in zwei Komponenten $K_{1}$ und $K_{2}$. Ist $\varepsilon$ eine positive Zahl, so gibt es ein einfach geschlossenes orientiertes Polygon $P^{\prime}$ mit folgenden Eigenschaften: (a) $P^{\prime} \subseteq K_{\mathbf{1}}$; (b) $\left\langle P^{\prime}\right\rangle$ $=\langle P\rangle ;(c)$ ist $U_{\theta}\left(F^{\prime}\right)$ die $\varepsilon$-Umgebung von $F$, so gilt $P^{\prime} \cap\left(K_{1} \backslash U_{\theta}\left(F^{\prime}\right)\right)$ $=P \cap\left(K_{1} \backslash U_{\varepsilon}(F)\right)$.

Um das zu beweisen, bemerke man zunächst, daß es einen Homöomorphismus von $E^{3}$ auf sich gibt, der die abgeschlossene Hülle $\bar{K}_{2}$ von $K_{2}$ auf einen Würfel und $F$ auf eine Seite dieses Würfels abbildet (siehe [8], Satz 1, Seite 22). Man braucht die Behauptung also nur unter der Voraussetzung zu beweisen, daß $\bar{K}_{2}$ ein Würfel und $F$ eine Seite von $\bar{K}_{2}$ ist. In diesem Falle ist der Beweis jedoch ganz einfach.

(B) Es sei $C$ eine zahme in $B^{3} \backslash A$ gelegene einfach geschlossene orientierte Kurve, die mit einem Polygon $L_{k_{0}}^{i_{0}}$ verschlungen ist. Dann gilt $\varkappa_{i_{0}} \leqslant\langle C\rangle$.

Beim Beweis dürfen wir voraussetzen, daß für $i>i_{0}$ stets $\varkappa_{i} \nless\langle C\rangle$ gilt, denn aus $x_{i} \leqslant\langle\boldsymbol{C}\rangle$ und $x_{i_{0}}<x_{i}$ folgt ja sofort $x_{i_{0}} \leqslant\langle\boldsymbol{C}\rangle$. Außerdem dürfen wir voraussetzen, daß $C$ ein Polygon ist, dessen Eckpunkte nur irrationale Koordinaten besitzen (siehe Abschn. 9).

Wir definieren vier Mengen $\mathfrak{P}_{1} \supseteq \mathfrak{P}_{\mathbf{2}} \supseteq \mathfrak{P}_{3} \supseteq \mathfrak{P}_{4}$ einfach geschlossener orientierter Polygone:

$\mathfrak{P}_{1}$ ist die Menge aller orientierten einfach geschlossenen Polygone $\boldsymbol{P}$ - in $E^{3} 、 A$, deren Eckpunkte nur irrationale Koordinaten besitzen und für die $\langle P\rangle=\langle C\rangle$ und $\mathfrak{v}\left(P, L_{k_{0}}^{i_{0}}\right)=1$ gilt.

Zu jedem $P$ aus $\mathfrak{P}_{1}$ gibt es eine größte Zahl $n(P)$, für die $P \cap A_{\boldsymbol{n}(\boldsymbol{P})} \neq \mathbf{0}$ ist. Es sei $n=\min n(P)\left(P \in \mathfrak{P}_{1}\right)$. Da $P$ mit dem Polygon $L_{k_{0}}^{i_{0}}$ verschlungen ist, muß $P$ das von $I_{k_{0}}^{i_{0}}$ berandete Quadrat $D_{k_{0}}^{i_{0}}$ schneiden, und $n$ kann nicht kleiner als $i_{0}$ sein.

$\mathfrak{P}_{2}$ ist die Menge aller $P$ aus $\mathfrak{P}_{1}$ mit $n(P)=n$.

Ist $P$ aus $\mathfrak{P}_{2}$, so gilt $P \cap A_{n} \neq 0, P \cap A_{n+1}=0$, so da $P$ einen der Würfel $W_{k}^{n}$ schneiden muß. Mit $m(P)$ sei die Anzahl dieser von $\boldsymbol{P}$ geschnittenen Würfel $W_{k}^{n}$ bezeichnet. Wir setzen $m=\min m(P)\left(P \in \mathfrak{P}_{2}\right)$.

$\mathfrak{P}_{3}$ sei die Menge aller $P$ aus $\mathfrak{P}_{2}$ mit $m(P)=m$.

Nun wählen wir irgendeinen der Würfel $W_{k}^{n}$, die von einem zu $\mathfrak{P}_{3}$ gehörenden Polygon geschnitten werden, aus und bezeichnen ihn mit $W$.

$\mathfrak{P}_{4}$ ist dann die Menge aller $P$ aus $\mathfrak{P}_{3}$ mit $P \cap W \neq 0$.

Man sieht sofort, daß die Mengen $\mathfrak{P}_{1}, \mathfrak{P}_{2}, \mathfrak{P}_{3}$ and $\mathfrak{P}_{4}$ nicht leer sind. Wie schon oben bemerkt gilt außerdem $n \geqslant i_{0}$. Wir wollen jetzt die Gleichung $n=i_{0}$ beweisen. Das soll indirekt geschehen. Wir nehmen also $n>i_{0}$ an und leiten daraus einen Widerspruch her.

Die Zahl $k$ sei der Index, für den $W=W_{k}^{n}$ wird. Zur Abkürzung setzen wir $\nabla=\nabla_{k}^{n}, R=R_{k}^{n}$ und $D=D_{k}^{n}$. Mit $D^{\prime}$ bezeichnen wir den von $D$ verschiedenen Deckel der Röhre $R$. Ist $P$ ein Polygon aus $\mathfrak{P}_{4}$, so gilt $0 \neq P \cap W \subseteq R$. Da $P$ die Menge $D_{k_{0}}^{i_{0}}$ schneidet, mu $P$ auch einen 
der Deckel $D$ oder $D^{\prime}$ schneiden, und da die Eckpunkte von $P$ nur irrationale Koordinaten besitzen, besteht der Durchschnitt $P \cap\left(D \cup D^{\prime}\right)$ aus endlich vielen Punkten. Es sei nun $P$ aus $\mathfrak{P}_{4}$ so gewählt, daß die Anzahl der Punkte in $P \cap\left(D \cup D^{\prime}\right)$ möglichst klein ist. Wir nehmen im folgenden an, daß $P$ den Deckel $D$ schneidet. Wird $D^{\prime}$ von $P$ geschnitten, so verläuft der Beweis ganz analog.

Aus $n>i_{0}$ folgt (nach der am Anfang des Beweises gemachten: Voraussetzung) $x_{n} \nless\langle C\rangle=\langle P\rangle$. Da die Röhre $R$ (bei passender Orientierung) in $W$ den Knoten $\varkappa_{n}$ repräsentiert, gibt es wegen der Bemerkung (A) aus Abschn. 10 ein in $E^{3} \backslash V$ gelegenes zur Vollkugel homöomorphes Polyeder $K$, das $P$ im Innern enthält. Der Rand von $K$ sei mit $S^{2}$ bezeichnet. Wir denken $K$ so gewählt, daß die Eckpunkte von $S^{2}$ (bei einer passenden Triangulierung) nur irrationale Koordinaten besitzen. Hieraus folgt, daß $S^{2} \cap D$ aus endlich vielen paarweise disjunkten einfach geschlossenen Polygonen besteht. Wir denken $K$ zudem so gewählt, daß die Anzahl der Komponenten von $S^{2} \cap D$ möglichst klein wird. Aus $D \cap P \neq 0$ folgt sofort $D \cap S^{2} \neq 0$. Mit $J$ bezeichnen wir eine Komponente von $D \cap S^{2}$, die so gewählt ist, daß die von $J$ berandete einfach zusammenhängende Komponente $I$ von $D \backslash J$ keinen Punkt von $S^{2}$ enthält. Die Menge $F=I \cup J$ ist dann ein zur Kreisscheibe homöomorphes Polyeder mit der Randkurve $J$. Aus der Tatsache, daß $S^{2} \cap D$ aus möglichst wenigen Komponenten besteht, folgt leicht, daß $I$ im Innern von $K$ liegt und $F \cap P$ nicht leer ist. Es seien $K_{1}$ und $K_{2}$ die beiden Komponenten von $K \backslash F$ und $P_{i}=P \cap K_{i}(i=1,2)$. Da $P$ mit $L_{k_{0}}^{i_{0}}$ verschlungen ist, muß $P$ mit dem von $L_{k_{0}}^{i_{0}}$ berandeten Quadrat $D_{k_{0}}^{i_{0}}$ (das wegen $n>i_{0}$ zu $D$ fremd ist) eine ungerade Anzahl von Punkten gemeinsam haben (da die Koordinaten der Eckpunkte von $P$ irrational sind, liegt kein Eckpunkt von $P$ auf der $D_{k_{0}}^{i_{0}}$ enthaltenden Ebene). Es muß also für einen Index $i=1,2$ die Menge $P_{i} \cap D_{k_{0}}^{i_{0}}$ aus einer ungeraden Anzahl von Punkten bestehen. Wir denken die Bezeichnungen so gewählt, daß das für $i=1$ eintritt. Die positive Zahl $\varepsilon$ sei so klein gewählt, daß die $\varepsilon$-Umgebung $U_{s}(F)$ von $F^{i}$ zu $D_{k_{0}}^{i_{0}}$ fremd ist, in $E^{3} \backslash A_{n+1}$ liegt und die Bedingung $\boldsymbol{U}_{\mathrm{a}}(\boldsymbol{F}) \cap \boldsymbol{D} \cap \boldsymbol{K}=\boldsymbol{F}$ erfüllt. Nun wenden wir die im Teil (A) dieses Beweises bewiesene Behauptung an und erhalten so ein einfach geschlossenes Polygon $P^{\prime}$, das man offenbar so wählen kann, daß alle seine Eckpunkte nur irrationale Koordinaten besitzen. $\mathrm{Da} U_{s}(F)$ zu $D_{k_{0}}^{i_{0}}$ fremd ist, gilt $P^{\prime} \cap D_{k_{0}}^{i_{0}}=P_{1} \cap D_{k_{0}}^{i_{0}}$, so daß $P^{\prime}$ mit $L_{k_{0}}^{i_{0}}$ verschlungen ist. Außerdem gilt $P^{\prime} \subseteq E^{3} \backslash A_{n+1}$, und $P^{\prime}$ schneidet nur solche Würfel $W_{k}^{n}$, die auch von $P$ geschnitten werden. Daraus folgt $P^{\prime} \in \mathfrak{P}_{4}$. Da beim Utbergang von $P$ zu $P^{\prime}$ die in $F$ gelegenen Schnittpunkte von $P$ und $D$ verschwunden sind, neue Schnittpunkte wegen $U_{s}(F) \cap D \cap K=F$ nicht hinzukamen, muß $P^{\prime} \cap\left(D \cup D^{\prime}\right)$ weniger Punkte als $P \cap\left(D \cup D^{\prime}\right)$ enthalten, was einen Widerspruch zur Auswahl von $P$ darstellt.
Damit ist die Gleichung $n=i_{0}$ bewiesen. Es gibt also ein einfach geschlossenes orientiertes Polygon $P$ mit folgenden Eigenschaften: $P \subseteq E^{3} 、 A_{i_{0}+1} ;\langle P\rangle=\langle C\rangle ; \mathfrak{v}\left(P, L_{k_{0}}^{i_{0}}\right)=1$. Nun läßt sich der Beweis der Behauptung (B) rasch beenden; Wir betrachten den Würfel $W_{k_{0}}^{i_{0}}$, die Röhre $R_{k_{0}}^{i_{0}}$ und die Menge $V_{k_{0}}^{i_{0}}$. Die Röhre $R_{k_{0}}^{i_{0}}$ repräsentiert bei passender Orientierung in $W_{k_{0}}^{i_{0}}$ den Knoten $\varkappa_{i_{0}}$. Da $P$ in $E^{3} \backslash V_{k_{0}}^{i_{0}}$ liegt $\left(V_{k_{0}}^{i_{0}}\right.$ ist ja in $A_{i+1}$ enthalten) und mit $L_{k_{0}}^{i_{0}}$ verschlungen ist, braucht man nur die Bemerkung (B) aus Abschn. 10 anzuwenden, um das gewünschte Resultat $\boldsymbol{x}_{i_{0}} \leqslant\langle P\rangle=\langle C\rangle$ zu erhalten.

Mit diesem Beweis ist die im Anschluß an den Hilfssatz 3 gemachte Bemerkung bereits bewiesen, denn für $i_{0}>1$ ist $\varkappa_{i_{0}}$ sicher nicht der triviale Knoten (es gilt ja $\varkappa_{i_{0}-1}<\varkappa_{i_{0}}$ ).

(C) Für jeden Index $i$ kann man aus der Menge $\mathfrak{L}_{0}^{i}$ aller Zyklen $L_{k}^{i}(1 \leqslant k \leqslant r(i))$ eine Teilmenge $\mathfrak{L}^{i}$ so auswählen, daß für jeden Index $n \geqslant 2$ die Menge $\Omega^{1} \cup \ldots \cup \Omega^{n-1}$ eine Homologiebasis von $A_{n}$ bildet.

Beweis. Wir betrachten das Polyeder

$$
A_{n}^{*}=A_{n} \cup \underset{\substack{1 \leqslant i<n \\ 1 \leqslant k \leqslant r(i)}}{\bigcup} D_{k}^{i}
$$

das aus $A_{n}$ durch Vereinigung mit allen nicht schon in $A_{n}$ enthaltenen Quadraten $D_{k}^{i}$ hervorgeht. Man sieht leicht, daß die beschränkten Komponenten von $E^{3} \backslash A_{n}^{*}$ zur offenen Vollkugel homöomorph sind, während die unbeschränkte Komponente von $E^{3} \backslash A_{n}^{*}$ zum Komplement einer abgeschlossenen Vollkugel homöomorph ist, so daß die eindimensionale Homologiegruppe von $E^{\natural} \backslash A_{n}^{*}$ nur aus dem Nullelement besteht. Hieraus ergibt sich, daß jeder in $E^{\mathbf{3}} \backslash A_{n}$ gelegene und in dieser Menge nicht nullhomologe Zyklus mit einem der Zyklen $L_{k}^{i}(1 \leqslant i<n ; 1 \leqslant k \leqslant r(i))$ verschlungen sein muß. Aus dem was in Abschnitt 2 über duale Basen gesagt

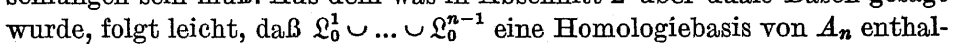
ten muß. $D$ as gilt insbesondere für $n=2$, so daß wir aus $\Omega_{0}^{1}$ eine Homologiebasis $\mathfrak{Q}^{1}$ von $A_{2}$ auswählen können. Indem wir nun benutzen, daß $\mathfrak{L}_{0}^{1} \cup \mathfrak{\Omega}_{0}^{2}$ eine Homologiebasis von $A_{3}$ enthält, können wir $\mathfrak{L}^{1}$ durch eine Teilmenge $\mathfrak{\Omega}^{2}$ von $\Omega_{0}^{2}$ zu einer Homologiebasis von $A_{3}$ ergänzen. So fortfahrend erhalten wir sukzessive die Mengen $\mathfrak{L}^{i}$ mit den geforderten Eigenschaften.

Wir kommen nun zum Beweis des Hilfssatzes 3. Dieser Beweis wird geführt sein, wenn wir zeigen können, daß jede der Kurven $C_{1}, \ldots, C_{t}$ in $E^{3} \backslash A\left(x^{*}\right)$ zu einem in $E^{3} \backslash A\left(x_{s}^{*}\right)$ enthaltenen Zyklus homolog ist, denn nach dem Dualitätssatz ist die Ordnung von $H\left(E^{\mathrm{B}} \backslash A\left(\varkappa_{s}^{*}\right)\right)$ gleich der Ordnung von $H\left(A\left(x_{s}^{*}\right)\right)$ also gleich $h\left(x_{s}^{*}\right)$ und damit kleiner als $t$. Es sei $m$ eine Zàhl, für die alle Kurven $C_{1}, \ldots, C_{t}$ in $E^{\mathbf{3}} \backslash A\left(\varkappa_{m}^{*}\right)$ liegen. Dabei dürfen wir $m>s$ annehmen. Aus (B) schließen wir, daß für $i>s$ keiner 
der Zyklen $C_{1}, \ldots, C_{t}$ mit einem Zyklus $L_{k}^{i}(1 \leqslant k \leqslant r(i))$ verschlungen ist. Wir betrachten nun die in (C) konstruierte Homologiebasis $\mathfrak{Q}^{1} \cup \ldots \cup \mathfrak{Q}^{s} \cup$ $\cup \mathfrak{L}^{s+1} \cup \ldots \cup \mathfrak{Q}^{m}$ von $A\left(x_{m}^{*}\right)=A_{m+1}$. Der erste Abschnitt $\mathfrak{L}^{1} \cup \ldots \cup \mathfrak{S}^{s}$ ist eine Homologiebasis von $A_{s+1}=A\left(x_{s}^{*}\right)$. Da keiner der Zyklen $C_{1}, \ldots, C_{t}$ mit einem Zyklus aus $\mathfrak{L}^{s+1} \cup \ldots \cup \mathfrak{Q}^{m}$ verschlungen ist, können wir unmittelbar die am Schluß des Abschnittes 2 gemachte Bemerkung anwenden und erhalten, daß die Zyklen $C_{1}, \ldots, C_{t}$ in $E^{3} \backslash A\left(\varkappa_{m}^{*}\right)$ und damit erst recht in $E^{3} \backslash A\left(\varkappa^{*}\right)$ zu Zyklen aus $E^{3} \backslash A\left(x_{s}^{*}\right)$ homolog sind.

14. Unterteilungen von Röhren. Wir betrachten eine Röhre $R$ im Einheitswürfel $W_{e}$ mit dem zugehörenden Komplex $\Re=\left\{W_{1}, \ldots, W_{r}\right\}_{\ell}$ dessen Würfel so numeriert seien, daß jeweils $W_{i}$ und $W_{i+1}$ eine gemeinsame Seite haben $(i=1, \ldots, r-1)$. Es sei $\Re$ ein $\nu$-Komplex. Wir betrachten ein Vielfaches $\varrho=\mu \cdot \nu$ von $v$. Unser Ziel ist es, die Röhre $R$ in einfacher Weise in $\mu^{2}$ Röhren zu zerlegen, deren zugehörende Komplexe $\varrho$-Komplexe sind und die $R$ durchlaufen, d.h. die beiden Deckel von $R$ miteinander verbinden. Dazu bezeichnen wir die gemeinsame Seite $W_{i} \cap W_{i+1}$ von $W_{i}$ und $W_{i+1}$ mit $S_{i}$ und den in $W_{1}$ bzw. $W_{r}$ enthaltenen Deckel von $R$ mit $S_{0}$ bzw. $S_{r}$. In $\left\{W_{e}\right\}^{\left({ }^{(\rho)}\right.}$ zerfällt jedes der Quadrate $S_{i}$ in $\mu^{2}$ Teilquadrate der Kantenlänge 1/e. Die Mittelpunkte dieser Teilquadrate, in die $S_{i}$ zerfällt, seien mit $m_{j}^{i}$ bezeichnet $(i=0, \ldots, r$; $\left.j=1, \ldots, \mu^{2}\right)$. Falls $S_{i-1}$ und $S_{i}$ nicht gegenüberliegende Seiten von $W_{i}$ sind, falls also $S_{i-1} \cap S_{i}$ eine Kante $K_{i}$ von $W_{i}$ ist, so betrachten wir die durch $K_{i}$ und die gegenüberliegende Kante von $W_{i}$ gehende Ebene. Diese Ebene schneidet $W_{i}$ in einem Rechteck, das $W_{i}$ diagonal zerlegt. Auf diesem Rechteck liegen genau $\mu^{2}$ Mittelpunkte $z_{j}^{i}\left(j=1, \ldots, \mu^{2}\right)$ von $z u\left\{W_{e}\right\}^{(e)}$ gehörenden Würfeln. Wir denken die Numerierung der Punkte $m_{j}^{i}$ und $z_{j}^{i}$ so vorgenommen, daß, falls $S_{i-1} \cap S_{i}=0$ ist, die Strecken $\left[m_{j}^{i-1}, m_{j}^{i}\right]$ auf $S_{i-1}$ und $S_{i}$ senkrecht stehen und, falls $S_{i-1} \cap S_{i} \neq 0$ ist, die Strecken $\left[m_{j}^{i-1}, z_{j}^{i}\right]$ auf $S_{i-1}$ und die Strecken $\left[z_{j}^{i}, m_{j}^{i}\right]$ auf $S_{i}$ senkrecht stehen. Wir setzen $T_{j}^{i}=\left[m_{j}^{i-1}, m_{j}^{i}\right]$ im ersten Fall und $T_{j}^{i}=\left[m_{j}^{i-1}, z_{j}^{i}\right] \cup$ $\cup\left[z_{j}^{i}, m_{j}^{i}\right]$ im zweiten Fall. Die Vereinigung aller Würfel aus $\left\{W_{e}\right\}^{(e)}$, die den Streckenzug $T_{j}=\bigcup_{i=1}^{r} T_{j}^{i}$ schneiden, ist eine Röhre $R_{j}$ von $W_{e}$ mit der Mittellinie $T_{j}$. Die $\underset{\text { zu }}{i=1}$ den $R_{j}$ gehörenden Komplexe $\Re_{j}$ sind $\varrho$-Komplexe und bestehen aus höchstens $2 \mu r$ Würfeln (jeder Würfel $W_{i}$ aus $\Re$ enthält höchstens $2 \mu$ Würfel aus $\Re_{\jmath}$ ). Wir wollen das System $R_{1}, \ldots, R_{\mu^{2}}$ die $\mu$-Unterteilung der Röhre $R$ nennen. Man sieht leicht, daß mit einer Röhre $R$ auch alle Röhren ihrer $\mu$-Unterteilung in $W_{e}$ unverknotet sind.

Ist $O$ eine einfach geschlossene Kurve im Innern von $W_{e}$ und $B$ eine zu $O$ fremde Sehne von $W_{e}$, so heißt $B$ mit $C$ verschlungen, falls für einen auf der Oberfläche von $W_{e}$ gelegenen Bogen $B^{*}$, der $B$ zu einem einfach geschlossenen Polygon $B \cup B^{*}$ ergänzt, $\mathfrak{v}\left(B \cup B^{*}, C\right)=1$ wird. (Diese Definition hängt von $B^{*}$ und von der Orientierung der
Sehne $B$ nicht ab.) Ist $R$ eine Röhre von $W_{e}$ und die Kurve $C$ zu $R$ fremd, so heißt $R$ mit $C$ verschlungen, falls die Mittellinie $B$ von $R$ mit $C$ verschlungen ist. Ist $R$ mit $C$ verschlungen, so sind auch die Röhren der $\mu$-Unterteilung von $R$ mit $C$ verschlungen.

15. Beweis des Hauptsatzes. Wir haben zu zeigen, daß es zu jeder eindimensionalen abgeschlossenen Menge $\mathcal{A}$ eine zur Sierpińskischen Universalkurve homöomorphe Mienge $X$ gibt, die sich nicht lagetreu in $\boldsymbol{A}$ einbetten läßt. Dazu genügt es, zu jeder im Abschnitt 11 definierten Menge $A\left(\varkappa^{*} ; \mu^{*}\right)$ eine zur Sierpińskichen Universalkurve homöomorphe Menge $X$ zu konstruieren, die sich nicht lagetreu in $A\left(x^{*} ; \mu^{*}\right) \cap \operatorname{Int} W_{c}$ einbetten läßt (Int $W_{e}$ ist die Menge aller inneren Punkte des Einheitswürfels $W_{e}$ ). In der Tat, wegen des Satzes aus Abschnitt 3 dürfen wir $\boldsymbol{A}$ als kompakt voraussetzen, so daß sich $A$ nach dem Satz aus Abschnitt 12 für eine gewisse Menge $A\left(\varkappa^{*} ; \mu^{*}\right)$ in $A\left(\varkappa^{*} ; \mu^{*}\right) \cap$ Int $W_{e}$ lagetreu einbetten läßt. Ist dann $X$ nicht lagetreu in $A\left(\varkappa^{*} ; \mu^{*}\right) \cap$ Int $W_{e}$ einbettbar, so ist $X$ auch nicht lagetreu in $A$ einbettbar.

Es sei jetzt eine Menge $A\left(\varkappa^{*} ; \mu^{*}\right)$ fest vorgegeben. Dabei ist $\varkappa^{*}=\left(x_{1}, x_{2}, \ldots\right)$ eine Knotenfolge und $\mu^{*}=\left(\mu_{1}, \mu_{2}, \ldots\right)$ eine Folge positiver ganzer Zahlen. Die Zahlen $\nu_{i}$ seien so festgelegt, daß $\mathfrak{A}\left(\varkappa_{i}^{*} ; \mu_{i}^{*}\right)^{\left(\mu_{i+1}\right)}$ ein $\nu_{i}$-Komplex ist $(i=1,2, \ldots)$.

Es ist nicht schwer, schrittweise eine Folge $\lambda^{*}=\left(\lambda_{1}, \lambda_{2}, \ldots\right)$ von Knoten mit folgenden Eigenschaften zu konstruieren. (Man beachte dabei nur, daß das Produkt zweier nicht trivialer Knoten stärker verknotet ist als jeder der Faktoren.)

$$
\lambda_{i}<\lambda_{i+1} \quad(i=1,2, \ldots) .
$$

(b) $\mathrm{Zu}$ jedem Index $i$ gibt es einen Index $k>i$, so daß $\left(v_{k} / v_{i}\right)^{2}$ größer ist als die Ordnung $h\left(\lambda_{1}, \ldots, \lambda_{i}\right)$ der Homologiegruppe $H\left(A\left(\lambda_{1}, \ldots, \lambda_{i}\right)\right)$ und $\lambda_{i+1} \nless\left(x_{k+1} \varkappa_{k+1}^{\prime}\right)^{22_{i}^{2} \gamma_{k}}$ gilt, wobei $\varkappa_{k+1}^{\prime}$ der aus $x_{k+1}$ durch Umkehrung der Orientierung hervorgehende Knoten ist.

Für eine solche Folge $\lambda^{*}$ definieren wir $X$ durch

$$
X=A\left(\lambda^{*}\right) \text {. }
$$

$X$ ist eine zur Sierpińskischen Universalkurve homöomorphe Menge (siehe Abschn. 11). Es gilt jetzt zu zeigen, daß sich $X$ nicht lagetreu in $A\left(x^{*} ; \mu^{*}\right) \frown$ Int $W_{e}$ einbetten läßt. Diesen Beweis führen wir indirekt. Wir nehmen also an, $h$ sei ein Homöomorphismus von $E^{3}$ auf sich, der $X$ in die Menge $A\left(\varkappa^{*} ; \mu^{*}\right) \frown$ Int $W_{e}$ abbildet. Nach der Bemerkung im Anschluß an den Hilfssatz 3 aus Abschnitt 13 gibt es in $h(X)$ eine zahme einfach geschlossene Kurve $L$ mit der Eigenschaft, daß jede in $E^{\text {s }} \backslash h(X)$ gelegene zahme einfach geschlossene orientierte Kurve, die mit $L$ ver- 
schlungen ist, verknotet sein muß. Es sei $L$ eine fest gewählte Kurve mit dieser Eigenschaft. Den Widerspruch zur angenommenen Existenz von $h$ werden wir erhalten, indem wir ein unverknotetes in $B^{\mathbf{3}} \backslash h(X)$ gelegenes mit $L$ verschlungenes einfach geschlossenes Polygon konstruieren.

Da $L$ im Innern von $W_{e}$ liegt, ist die Vereinigung von $L$ mit der Oberfläche des Würfels We zahm (siehe z.B. [12] Satz 8.1), und es gibt einen Homöomorphismus $g$ von $E^{3}$ auf sich, der alle nicht in $W_{e}$ gelegenen Punkte festläßt und $L$ auf ein Polygon $L_{0}$ abbildet. Man findet dann leicht eine unverknotete mit $I_{0}$ verschlungene Sehne $B_{0}$ von $W_{c}$ (siehe Abschn. 14). Da $g^{-1}\left(B_{0}\right)$ von $L$ einen positiven Abstand hat, folgt aus [11] Satz 1 und [10] Satz $1, \mathrm{da} B$ es in $W_{e}$ eine unverknotete mit $L$ verschlungene Sehne gibt (es sei daran erinnert, daß Sehnen stets Polygone sind). Hierauf ist es nicht schwer, für genügend großes $v$ eine mit $L$ verschlungene unverknotete Röhre von $W_{e}$ zu finden, deren Komplex ein $v$-Komplex ist. Es gibt also einen Index $i$ und eine unverknotete mit $L$ verschlungene Röhre $R$ von $W_{e}$, deren Komplex ein $\nu_{i}$-Komplex ist. Einen solchen Index $i$ und eine solche Röhre $R$ denken wir fest ausgewählt. $D$ und $D^{\prime}$ seien die Deckel von $R$. Mit $k$ bezeichnen wir einen Index, für den bei diesem $i$ die Eigenschaft (b) der Folge $\lambda^{*}$ erfüllt ist. Sodann betrachten wir die $\left(v_{k} / v_{t}\right)$-Unterteilung der Röhre $R$ (siehe Abschn. 14). Die dabei auftretenden Röhren seien mit $R_{3}$ bezeichnet $\left(j=1, \ldots,\left(v_{k} \mid v_{i}\right)^{2}\right)$. Es gilt dann:

(a) Die zu den $R_{j}$ gehörenden Komplexe $\Re_{j}$ sind $v_{k}$-Komplexe.

(b) Die $R_{f}$ sind in $W_{e}$ unverknotet.

(c) Die $R_{j}$ sind mit $L$ verschlungen.

(d) Zu jeder Röhre $R_{j}$ gibt es eine zu $A\left(\varkappa^{*} ; \mu^{*}\right)$ fremde in $D$ beginnende und in $D^{\prime}$ endende Sehne $B_{j}$ von $W_{e}$, deren Endpunkte die beiden Mittelpunkte der Deckel von $R_{f}$ sind, die mit Ausnahme ihrer Endpunkte im Innern von $R_{j}$ verläuft und die in $W_{e}$ einen Knoten $\left(x_{k+1} \cdot x_{k+1}^{\prime}\right)^{\beta_{j}}$ mit $\beta_{j}<2 \nu_{i}^{2} \nu_{k}$ repräsentiert.

Die Eigenschaften (a), (b) und (c) folgen unmittelbar aus den im Abschnitt 14 angegebenen Eigenschaften der Unterteilungen von Röhren. Es braucht also nur (d) bewiesen zu werden. Dazu betrachten wir eine Röhre $R_{f}$, die wir so orientiert denken, daß ihr Eingang im Deckel $D$ von $R$ enthalten ist. $\Re_{j}=\left\{W_{1}, \ldots, W_{s}\right\}$ sei der zu $R_{j}$ gehörende Komplex, dessen Wüirfel in der natürlichen Reihenfolge angeordnet seien. Es sei $S_{l}=W_{l} \cap W_{l+1}(l=1, \ldots, s-1)$, während $S_{0}$ und $S_{s}$ die in $D$ bzw. $D^{\prime}$ enthaltenen Deckel von $R_{\jmath}$ seien. Da $\Re$ weniger als $\nu_{i}^{\mathbf{3}}$ Würfel enthält, gilt $\left.s<2 v_{i}^{8}\left(v_{k}\right) v_{i}\right)=2 v_{i}^{2} v_{k}(\Re$ ist der Komplex von $R$; siehe
Abschn. 14). Ist $m_{l}$ der Mittelpunkt von $S_{l}(l=0,1, \ldots, s)$, so gibt es in $W_{l}$ eine von $m_{l-1}$ nach $m_{l}$ verlaufende zu $A\left(x^{*} ; \mu^{*}\right)$ fremde Sehne $C_{l}$, die in $W_{l}$ den Knoten $\varkappa_{k+1} \cdot \varkappa_{k+1}^{\prime}$ repräsentiert (es ist ja $W_{l} \cap A\left(x_{k+1}^{*} ; \mu_{k+1}^{*}\right)$ $\subseteq W_{l}\left(\varkappa_{k+1}\right)$; vgl. die Bemerkung (A) aus Abschn. 11). Die Vereinigung $B_{j}=\bigcup_{l=1}^{s} C_{l}$ ist dann eine Sehne von $W_{e}$, die alle in (d) geforderten Eigenschaften besitzt (vgl. die Bemerkung (C) aus Abschn. 10).

Es seien $a_{j}$ bzw. $b_{j}$ die auf den Deckeln $D$ bzw. $D^{\prime}$ von $R$ gelegenen Endpunkte von $B_{j}$. Wir verbinden jeden der Punkte $a_{j}$ und $b_{j}$ durch einen auf der Oberfläche von $W_{e}$ verlaufenden Streckenzug mit dem Punkt $o=(0,0,0)$, wobei wir darauf achten, daß je zwei dieser Streckenzüge nur den Punkt $o$ gemeinsam haben. Für $1 \leqslant j \leqslant\left(v_{k} / v_{i}\right)^{2}$ sei dann $J_{j}$ das aus $B_{j}$ und den $a_{j}$ bzw. $b_{j}$ mit $o$ verbindenden Streckenzügen bestehende einfach geschlossene Polygon, das durch $B_{j}$ auf natürliche Weise orientiert ist. Jedes dieser Polygone $J_{j}$ repräsentiert einen Knoten $\left(\varkappa_{k+1} \cdot \varkappa_{k+1}^{\prime}\right)^{\beta_{j}}$ mit $\beta_{j}<2 \nu_{i}^{2} \nu_{k}$. Berücksichtigt man, daß hieraus wegen der Wahl von $k$ sofort $\lambda_{i+1} \nless\left\langle J_{j}\right\rangle$ folgt und die Anzahl der Polygone $J_{\xi}$ größer als $h\left(\lambda_{1}, \ldots, \lambda_{i}\right)$ ist, so ergibt sich aus der Tatsache, daß die Polygone $J_{j} \mathrm{zu} A\left(x^{*} ; \mu^{*}\right) \cap$ Int $W_{e}$ also erst recht $\mathrm{zu} h(X)$ fremd sind, durch Anwendung des Hilfssatzes 3 aus Abschnitt 13, daß zwei der Polygone $J_{\text {, }}$ in $E^{3} \backslash h(X)$ homolog sein müssen. Es sei etwa $J_{1} \mathrm{zu} J_{2}$ homolog. Mit $x$ bezeichnen wir eine zweidimensionale Kette, deren Rand der Zyklus $J_{1}+J_{2}$ ist ( $x$ sei in $E^{3} \backslash h(X)$ gelegen). Aus $J_{1} \cap J_{2}=\{0\}$ folgt, daß die Vereinigung $\bar{x}$ aller zu $x$ gehörenden Dreiecke $J_{1} \cup J_{2}$ enthält. Natürlich sind $J_{1}$ und $J_{2}$ im Polyeder $\bar{x}$ homolog.

Wir zeigen nun, daß der Durchschnitt von $\bar{x}$ mit dem Mantel $\boldsymbol{M}$ der $J_{1}$ enthaltenden Röhre $R_{1}$ einen die beiden Deckel von $R_{1}$ verbindenden Streckenzug $C$ enthalten muß. (Unter dem Mantel von $R_{1}$ verstehen wir die Menge der Randpunkte von $R_{1}$, die nicht im Innern eines der Deckel von $R_{1}$ liegen.) Es genügt offenbar zu zeigen, daß $M \cap \bar{x}$ eine Komponente besitzt, die beide Deckel von $R_{1}$ schneidet. Gäbe es keine solche Komponente, so könnte man auf $M \frown \operatorname{Int} W_{e}$ eine einfach geschlossene Kurve $K$ finden, die zu $\bar{x}$ fremd ist und die $R_{1}$ genau einmal umschlingt. Es gälte dann $\mathfrak{v}\left(K, J_{1}\right)=1$ und $\mathfrak{v}\left(K, J_{2}\right)=0$, was unmöglich ist, da $J_{1}$ und $J_{2}$ in $\bar{x}$ also in $E^{3} \backslash K$ homolog sind.

Orientiert man den Streckenzug $C$ irgendwie, so erhält man eine Sehne von $W_{e}$, die nach der Bemerkung (D) aus Abschnitt 10 unverknotet ist. Da $R_{1}$ mit $L$ verschlungen ist, mu $\beta$ auch $C$ mit $L$ verschlungen sein, so daß man, indem man $C$ durch einen auf der Oberfläche von $W_{e}$ gelegenen Bogen zu einem einfach geschlossenen Polygon ergänzt, ein in $E^{3} \backslash h(\boldsymbol{X})$ gelegenes unverknotetes einfach geschlossenes Polygon erhält, das mit $L$ verschlungen ist. Das ist der bereits oben angekündigte Widerspruch, und der Hauptsatz ist bewiesen. 


\section{Literaturverzeichnis}

[1] P. Alexandroff, Dimensionstheorie, Math. Ann. 106 (1932), S. 161-238.

[2] - und H. Hopf, Topologie I, Berlin 1935.

[3] R. D. Anderson, One-dimensional continuous curves and a homogeneity theorem, Ann. of Math. 68 (1958), S. 1-16.

[4] R. H. Bing, Approximating surfaces with polyhedral ones, Ann. of Math. 65 (1957), S. 456-483.

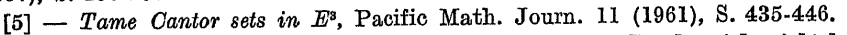

[6] H. G. Bothe, Ein eindimensionales Kompaltum im $E^{s}$, das sich nicht lagetreu in die Mengersche Universalkurve einbetten läßt, Fund. Math. 54 (1964), S. 251-258.

[7] - Universalmengen bezüglich der Lage im $E^{n}$, Fund. Math. 56 (1965), S. 203-212.

[8] W. Graeub, Die semilinearen Abbildungen, Sitzungsber. d. Heidelberger Akad. d. Wiss. 1950, 4. Abhandl.

[9] C. Kuratowski, Topologie II, Warszawa 1952.

[10] E. E. Moise, Affine structures in 3-manifolds II, Ann. of Math. 55 (1952), S. 172-176.

[11] - Affine structures in 3-manifolds IV, Ann. of Math. 55 (1952), S. 215-222.

[12] - Affine structures in 3-manifolds VIII, Ann. of Math. 59 (1954), S. 159-170

[13] H. Schubert, Die eindentige Zerlegbarkeit eines Knotens in Primknoten. Sitzungsber. d. Heidelberger Akad. d. Wiss. 1949, 3. Abhandl.

[14] - Knoten und Vollringe, Acta Math. 90 (1953), S. 131-286.

Reçu par la Rédaction le 29.6.1964

\section{Generalized Fréchet varieties}

by

J. S e g a l (Seattle, Wash.)

Introduction. The theory of Fréchet varieties and specifically Fréchet surfaces has been a subject of considerable interest not only in its own right but because of its applications to the theory of length and area. Though progress in this area had been made for over sixty years the more recent work of Cesari, Federer, Rado [4] and Youngs [7] has given it a sense of completeness. In the latter work the representation problem is solved for Fréchet surfaces.

This paper deals with the representation problem for two new collections of spaces, called monovarieties and perivarieties, which are each generalizations of the notion of Fréchet variety. In Section 1 we state the representation problem for monovarieties and consider the general situation. In Section 2 we obtain a reduction theorem for some cases of monoequivalence analogous to Youngs' reduction theorem for Fréchet equivalence. Then we show that a monosurface is actually a Fréchet surface. In Section 3 we state the representation problem for perivarieties and consider the general situation. In Section 4 we obtain a reduction theorem for periequivalence. Then we show that a perisurface is also a Fréchet surface. So since the representation problem has been solved for Fréchet surfaces, we have a solution for monosurfaces and perisurfaces.

We attempt to parallel Youngs' notation and development of Fréchet varieties. All manifolds considered are compact and connected.

\section{The representation problem for monovarieties.}

Notation. If $f_{n}: X \rightarrow Y$ is a mapping where $X$ and $Y$ are metric, $n=0,1,2, \ldots$, then the notation $f_{n} \Rightarrow f_{0}$ means that $f_{n}$ converges uniformly to $f_{0}$; that is, if $\varrho\left\{f_{n}, f_{0}\right\}=\sup \varrho\left\{f_{n}(x), f_{0}(x)\right\}, x \in X$, where $\varrho$ is the distance function in $\bar{Y}$, then $\bar{\varrho}\left\{f_{n}, f_{0}\right\} \rightarrow 0$. Also $f: X \Rightarrow \bar{Y}$ means that $f$ maps $X$ onto $Y$. Throughout this paper $\mathfrak{X}$ will denote the class of Peano spaces (i.e., the class of locally connected (metric) continua) and $f$ the class of mappings $f: X \rightarrow Y$ where $X \in \mathfrak{X}$ and $Y$ is metric. Whenever the

* This research was supported in part by NSF grant 24421; the author wishes to express his gratitude to Professor J. W. T. Youngs for his interest and assistance in the preparation of this paper. 